\title{
The Role of Arp2/3 in Growth Cone Actin Dynamics and Guidance Is Substrate Dependent
}

\author{
José E. San Miguel-Ruiz ${ }^{1,2}$ and Paul C. Letourneau ${ }^{1,2}$ \\ ${ }^{1}$ Departments of Neuroscience and ${ }^{2}$ Graduate Program in Neuroscience, University of Minnesota, Minneapolis, Minnesota 55455
}

\begin{abstract}
During development extrinsic guidance cues modulate the peripheral actin network in growth cones to direct axons to their targets. We wanted to understand the role of the actin nucleator Arp2/3 in growth cone actin dynamics and guidance. Since growth cones migrate in association with diverse adhesive substrates during development, we probed the hypothesis that the functional significance of Arp $2 / 3$ is substrate dependent. We report that Arp2/3 inhibition led to a reduction in the number of filopodia and growth cone F-actin content on laminin and L1. However, we found substrate-dependent differences in growth cone motility, actin retrograde flow, and guidance after Arp2/3 inhibition, suggesting that its role, and perhaps that of other actin binding proteins, in growth cone motility is substrate dependent.
\end{abstract}

Key words: actin; Arp2/3; growth cone; guidance; L1 CAM; laminin

\section{Introduction}

Developing axons are led by their terminal growth cones to the sites where they establish synaptic connections. Extrinsic guidance cues regulate the cytoskeletal machinery, particularly actin filaments, of growth cones to direct them toward their targets. Consequently, proper growth cone actin dynamics are crucial to correctly wire the nervous system (Bentley and ToroianRaymond, 1986; Lanier et al., 1999; Kwiatkowski et al., 2007; Gomez and Letourneau, 2013).

Arp2/3 is a frequently studied actin binding protein (ABP) and regulator of actin dynamics (Goley and Welch, 2006; Pollard, 2007). This multiprotein complex binds to the side of a preexisting actin filament and nucleates an actin branch (Mullins et al., 1997). This branched actin network has been implicated in numerous processes in non-neuronal cells (Goley and Welch, 2006), most commonly associated with lamellipodial formation and protrusion (Suraneni et al., 2012; Wu et al., 2012), though not all reports agree (Di Nardo et al., 2005). Arp2/3 is also required for haptotaxis in fibroblasts, but not chemotaxis (Wu et al., 2012); although, other groups have shown it is required for chemotaxis (Mukai et al., 2005; Suraneni et al., 2012). Additionally, Arp2/3 appears to influence filopodial numbers in cells (Ma-

\footnotetext{
Received Feb. 17, 2014; revised March 14, 2014; accepted March 19, 2014

Author contributions: J.E.S.M.-R. and P.C.L. designed research; J.E.S.M.-R. performed research; J.E.S.M.-R. and P.C.L. analyzed data; J.E.S.M.-R. and P.C.L. wrote the paper.

This project was funded by National Institutes of Health (NIH) grants HD019950 (P.C.L.), T32 GM 008244 Medical Scientist Training Program (J.E.S.M.-R.), 2T32 GM008470-18 (J.S.M.), and T32 DA022616 (J.S.M.).We thank Dr. Lorene Lanier for kindly providing the EGFP-CA and mCherry-CA peptide constructs; Dr. James R. Bamburg for kindly providing the mCherry-actin construct; the University Imaging Centers (http://uic.umn.edu) at the University of Minnesota for microscopes, technical advice, and assistance with imaging; Dr. Bonnie Marsick and Florence Roche for technical assistance; and Dr. Steven McLoon for technical advice and thoughtful discussions.

The authors declare no competing financial interests.

Correspondence should be addressed to Paul C. Letourneau, Department of Neuroscience, 6-145 Jackson Hall, University of Minnesota, 321 Church Street SE, Minneapolis MN 55455. E-mail:letou001@umn.edu.

DOI:10.1523/JNEUROSCI.0672-14.2014

Copyright $\odot 2014$ the authors $\quad 0270-6474 / 14 / 345895-14 \$ 15.00 / 0$
}

chesky and Insall, 1998; Svitkina et al., 2003), but not always (Sigal et al., 2007). Furthermore, it is linked to endocytosis and vesicular trafficking (Merrifield et al., 2004; Duleh and Welch, 2010). Last, Arp2/3 interacts with vinculin and FAK (DeMali et al., 2002; Serrels et al., 2007), influences integrin-based adhesions in fibroblasts (Wu et al., 2012), and promotes efficient cell adhesion between immune system cells (Butler and Cooper, 2009).

The role of Arp2/3 in growth cone actin dynamics and guidance is unclear. Initially, Arp2/3 was considered unnecessary for filopodial formation, actin organization in veils and in veil protrusion, and as a negative regulator of axon elongation, although guidance defects were reported when Arp2/3 was inhibited (Strasser et al., 2004). Later, Arp2/3 was reported to regulate filamentous actin (F-actin) content, veil protrusion and stability, and filopodial formation in growth cones (Korobova and Svitkina, 2008; Spillane et al., 2011; Yang et al., 2012). Additionally, Arp2/3 inhibition in Caenorhabditis elegans causes deficient axon guidance (Shakir et al., 2008; Norris et al., 2009). From these reports, Arp2/3 role in growth cone migration is still uncertain. We believe these discrepancies reflect different neuronal systems and in vitro conditions.

Growth cone morphology, behavior, and intracellular processes are influenced by the underlying substrate (Burden-Gulley et al., 1995; Liu et al., 2002). We used two naturally occurring substrates that have profound effects on growth cone morphology and behavior: the extracellular matrix protein laminin and the cell adhesion molecule (CAM) L1. On laminin, axons extend with compact filopodial growth cones, while on L1 growth cones are broad and lamellar. These differences could reflect influences that substrates have on ABPs in growth cones. Adhesion molecules are capable of signaling intracellularly and, therefore, influencing the activity of ABPs (Kamiguchi and Lemmon, 1997; Giancotti and Ruoslahti, 1999). Consequently, growth cone dependency on any given $\mathrm{ABP}$ could be determined by the substrate. We report here that Arp2/3 inhibition led to a reduction in 
the number of filopodia formed and F-actin content on laminin and L1. However, we found substrate-dependent differences in growth cone motility, retrograde actin flow, and guidance after Arp2/3 inhibition, suggesting that its role, and perhaps that of other ABPs, in growth cone motility is substrate dependent.

\section{Materials and Methods}

Materials. F-12 medium (21700-075), B27 (17504044), GlutaMAX (35050061), sodium pyruvate (11360-070), Antibiotic-Antimycotic (15240-062), Alexa Fluor 488- and 568-phalloidin, and Alexa Fluor 488 and 568 secondary antibodies were purchased from Life Technologies. Nerve growth factor (NGF; 256-GF-100/CF), Netrin (128-N1-025), ephrin-A2 (603-A2-200), and L1 (777-NC-100) were purchased from R\&D Systems. Chariot (30025) was purchased from Active Motif. CK666 (ALX-270-506) and cytochalasin D (BML-T109) were purchased from Enzo Life Sciences. Chicken neuron transfection kit was purchased from Lonza (VPG-1002). Fertilized White Leghorn chicken eggs were purchased from Hy-Line North America. Anti-ephrin A2 (sc-912), antiArp3 (sc-15390), and anti- $\beta 3$-Tubulin (sc-58888) antibodies were purchased form Santa Cruz Biotechnology Anti-p34 antibody was purchased from Millipore (07-227). Anti-8D9 (L1) antibody was purchased from the Developmental Studies Hybridoma Bank, University of Iowa. Anti-acetylated tubulin (32-2700) antibody was purchased from Life Technologies. All other reagents were acquired from Sigma-Aldrich, unless otherwise stated.

Neuronal culture. Coverslips were treated with $0.1 \%$ poly-L-lysine for $2 \mathrm{~h}$, washed three times in $\mathrm{H}_{2} \mathrm{O}^{\text {dd }}$, dried, and coated with $5 \%$ nitrocellulose dissolved in amyl acetate. Coverslips with dried nitrocellulose were coated overnight with either $20 \mu \mathrm{g} / \mathrm{ml}$ laminin or $4 \mu \mathrm{g} / \mathrm{ml} \mathrm{L1}$. Video dishes were assembled by gluing coverslips to Petri dishes with a $13 \mathrm{~mm}$ diameter hole on the bottom, UV sterilized overnight, then coated as above. Embryonic day 7 (E7) lumbar dorsal root ganglia (DRG) or retinal ganglion cell (RGC) neurons were cultured overnight as tissue explants or dissociated cells, in a humidified incubator at $40^{\circ} \mathrm{C}$, in accordance with the University of Minnesota Institutional Animal Care and Use Committee. Culture media was composed of F12H supplemented with $1 \times \mathrm{B} 27,2$ mm glutamine, $1 \mathrm{~mm}$ sodium pyruvate, $8 \mathrm{~mm}$ glucose, and $10 \mathrm{~mm}$ HEPES.

Neuronal transfection. A detailed protocol was described previously(Marsick et al., 2010, 2012). Approximately, $5 \times 10^{6} \mathrm{DRG}$ or RGC neurons were transfected with either $2 \mu \mathrm{g}$ of the EGFP or $2 \mu \mathrm{g}$ of EGFP-CA (construct prevents Arp2/3 activation by NWASP; kindly provided by Dr. Lorene Lanier, University of Minnesota, Minneapolis, MN). For retrograde flow measurements, $2 \mu \mathrm{g}$ mCherry-actin (kindly provided by Dr. James R. Bamburg, Colorado State University, Fort Collins, CO) was cotransfected with either EGFP or EGFP-CA. For study of integrin adhesions in growth cones, E7 DRG neurons were transfected with $2 \mu \mathrm{g}$ of paxillin-GFP purchased from Addgene (plasmid 15233). We transfected cells using a Lonza Nucleofector and the G13 program. Transfected cells were grown overnight with $0.5 \mathrm{ng} / \mathrm{ml} \mathrm{NGF}$.

Immunocytochemistry. A detailed protocol for fixation and blocking of neurons was described previously(Marsick et al., 2010, 2012). Antibodies were diluted in PBS with 10\% goat serum and 0.2\% Triton X-100. Antip34 (1:100), anti-Arp3 (1:100), anti-8D9 (L1) (1:100) anti-ephrin (1: $200)$, anti-acetylated tubulin (1:300), or anti- $\beta 3$-tubulin (1:500) were diluted in blocking media and applied for $4 \mathrm{~h}$. Coverslips were then washed three times in PBS and the corresponding secondary antibodies used: Alexa Fluor 568 goat anti-rabbit, Alexa Fluor 488 goat anti-mouse, or both were applied at a 1:500 dilution for $1 \mathrm{~h}$. If fluorescent phalloidin was used, it was applied with the secondary antibodies at a concentration of $2.5 \mu \mathrm{l}$ for every $100 \mu \mathrm{l}$ of blocking/antibody solution, either for the Alexa Fluor 488 or 568 phalloidin. Coverslips were washed three times in PBS and mounted with SlowFade (Invitrogen) and sealed.

Time-lapse microscopy, fluorescent quantification, and morphometric analysis. Image acquisition settings were held constant for the different groups within a particular experiment, and images were obtained in one session. All images were obtained using an Olympus XC-70 inverted microscope equipped with a Capital Q camera controlled by MetaMorph software (Molecular Devices), unless otherwise noted. Quantification of fluorescence intensity was done by measuring the integrated signal in the distal $40 \mu \mathrm{m}$ of the growth cone. Growth cone areas were obtained by thresholding of phalloidin-stained images and tracing growth cone outlines. Numbers of filopodia were obtained from phalloidin-labeled growth cones, and the number of filopodia per growth cone counted and divided by the growth cone perimeter length. This number was then expressed as the number of filopodia per $100 \mu \mathrm{m}$ of growth cone perimeter. Paxillin-GFP images were obtained using a Zeiss Observer Z1 TIRF microscope (University Imaging Centers) equipped with a QuantEM CCD.

Statistical analyses. Data were assessed for normality and variance and analyzed accordingly with an unpaired Student's $t$ test, Mann-Whitney $U$ test, ANOVA, or Kruskal-Wallis, based on the number of groups to be compared. Data are reported as mean \pm SEM.

Pharmacological inhibitors. Fifty micrometers of CK666 was added to cultures for $4 \mathrm{~h}$ previous to any manipulation (e.g., NGF stimulation or turning assays). Cytochalasin D was used at $2 \mu \mathrm{m}$. Control groups received the same volume of DMSO (dimethyl sulfoxide) that was used to deliver the drug treatment, without ever exceeding a $5 \mu \mathrm{l} / \mathrm{ml}$ concentration.

Retrograde flow measurements. To monitor the retrograde flow of actin in growth cones we transfected neurons with mCherry-actin. Arp $2 / 3$ inhibition was achieved pharmacologically with $50 \mu \mathrm{M}$ CK666 or by transfection of EGFP-CA; control groups received DMSO or EGFP transfection, respectively. Retrograde flow time lapses were acquired with a Zeiss Cell Observer Spinning Disk Confocal (University Imaging Centers) equipped with a QuantEM CCD with images obtained every $3 \mathrm{~s}$ for 3 min. Kymographs were obtained and analyzed in MATLAB, using a previously published algorithm developed in David Odde's Laboratory (Chan and Odde, 2008).

Barbed-end labeling. This protocol has been described in detail previously (Marsick et al., 2010).

Growth cone turning assay. This method was described previously (Marsick et al., 2010, 2012). Briefly, glass micropipettes were coated with $5 \%$ nitrocellulose in amyl acetate. These were then dipped in $1 \mu \mathrm{g} / \mathrm{ml}$ NGF in PBS for $3 \mathrm{~min}$. The coated micropipette was lowered into a video dish with a micromanipulator and placed $\sim 80 \mu \mathrm{m}$ away from the central domain of a growth cone at a $45^{\circ}$ angle, relative to the neurite axis. Growth cones were imaged every $30 \mathrm{~s}$. Turning responses were measured as the change in direction in growth cone migration after $45 \mathrm{~min}$ of presenting the NGF-coated micropipette.

Protein loading. The WASP VCA domain peptide was acquired from Cytoskeleton (VCG03-A) and reconstituted according to manufacturer's specifications. The VCA peptide was complexed with the Chariot reagent from Active Motif, based on manufacturer's specifications. After trying several ratios of the two components, we found that for our experiments the best concentrations were $4 \mu \mathrm{g}$ Chariot $+3 \mu$ VCA-GST incubated at room temperature for $30 \mathrm{~min}$. The complex was then delivered into growth cones from an immobilized nitrocellulose-coated pipette, as described (see above, Growth cone turning assay).

Stripe assay. Coverslips were dipped in a $0.5 \% 3$-aminopropyltrimethoxysilane solution in $\mathrm{H}_{2} \mathrm{O}^{\text {dd }}$ for half an hour, washed three times for $5 \mathrm{~min}$ in $\mathrm{H}_{2} \mathrm{O}^{\text {dd }}$, then air dried in a laminar flow hood, and coated with $5 \%$ nitrocellulose in amyl acetate. Silicon molds for the stripes were attached to the coverslips. The solution that passed through the channels was composed of $20 \mu \mathrm{g} / \mathrm{ml}$ ephrin A2-Fc and $10 \mu \mathrm{g} / \mathrm{ml}$ goat anti-Fc, diluted in either $20 \mu \mathrm{g} / \mathrm{ml}$ laminin or $4 \mu \mathrm{g} / \mathrm{ml} \mathrm{L1}$. A total volume of 250 $\mu \mathrm{l}$ of solution was passed though each silicon matrix over a period of $1 \mathrm{~h}$. At this point $50 \mu \mathrm{l}$ of PBS was passed through the channels to remove any unbound protein and the matrices were removed from the coverslips. Laminin $(20 \mu \mathrm{g} / \mathrm{ml}$ in PBS for $20 \mathrm{~min})$ or L1 $(4 \mu \mathrm{g} / \mathrm{ml}$ in PBS for $1 \mathrm{~h})$ was then applied to the entire coverslip. E7 temporal retina stripes were then plated over the stripes and grown for $16 \mathrm{~h}$. Next morning, 50\% of the media was replaced and either DMSO or $50 \mu \mathrm{M}$ CK666 was added to the cultures and allowed to grow for another $24 \mathrm{~h}$. At this point, the cultures were fixed, blocked, stained with anti-ephrin A2 and anti- $\beta 3$ tubulin antibody, and imaged. 

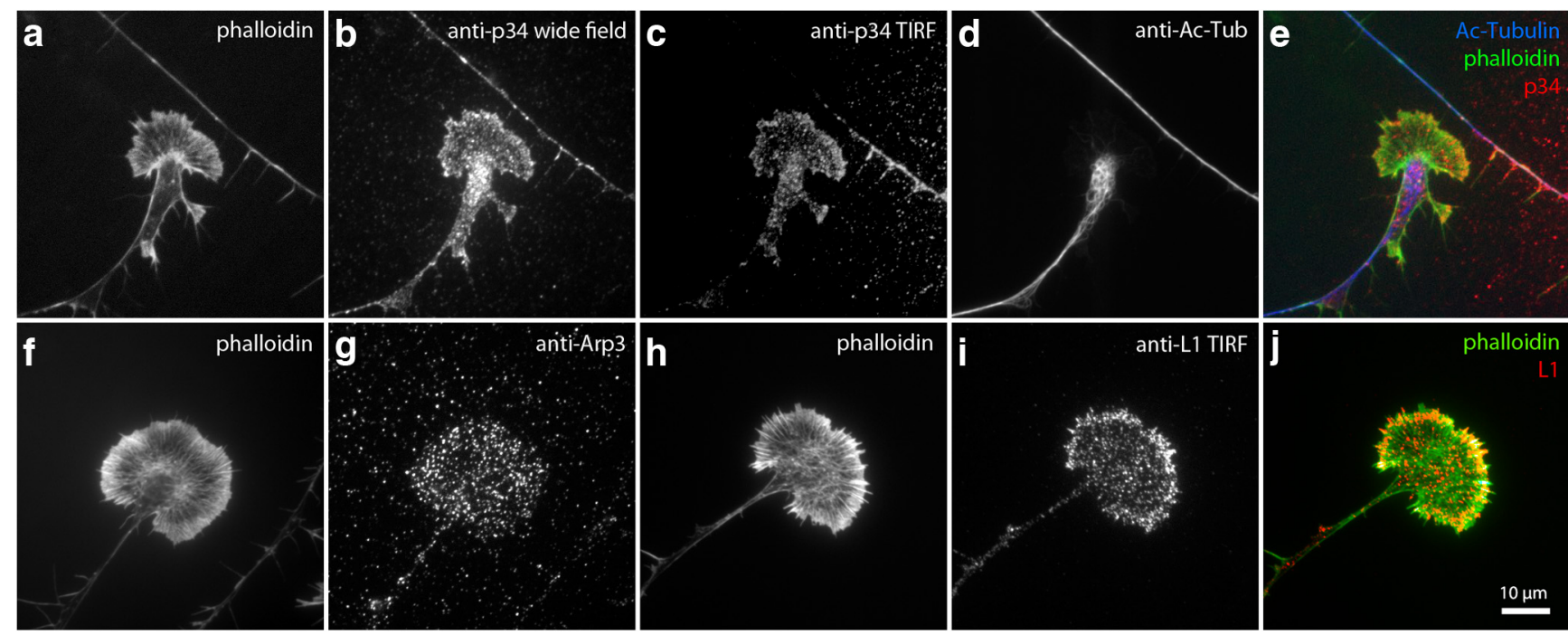

Figure 1. Arp2/3 is present in the leading edge of growth cones on L1. $a-j$, E7 DRG explants were grown overnight on L1, treated globally with $50 \mathrm{ng} / \mathrm{ml} \mathrm{NGF} \mathrm{for} 15 \mathrm{~min}$, fixed, and stained with phalloidin and antibodies against acetylated $\alpha$-tubulin (Ac-Tubulin), p34, Arp3, and L1 (8D9). Images were acquired with standard confocal microscopy; (c) p34 staining was also imaged with the TIRF modality to show its localization near the substrate.

\section{Results}

\section{L1 as a substrate}

The glycoprotein $\mathrm{L} 1$ is a homophilic binding CAM important for neurite growth, axon fasciculation and guidance, and synapse formation (Nakamura et al., 2010). As an in vitro substrate it promotes the formation of broad lamellar growth cones in E7 DRG or RGC neurons (Fig. 1a,f,h). These growth cones have broad, thin peripheral domains (P-domain) that are periodically subdivided by filopodia. These subdivisions are known as lamellipodial veils and are dependent on Arp2/3 function (Korobova and Svitkina, 2008).

\section{Arp2/3 at the leading edge}

To understand its role in actin dynamics and guidance, we first asked where Arp2/3 was localized in our growth cones. We performed immunostaining against two subunits of the Arp2/3 complex, p34 and Arp3. Consistent with previous reports, widefield microscopy showed the p34 subunit to be localized at the central domain (C-domain) and at the leading edge of growth cones (Fig. 1a,b). However, when we used total internal reflection fluorescence (TIRF) microscopy to image the cytoplasm near the substrate, most of the $\mathrm{C}$-domain staining was eliminated, but not the anti-p34 staining at leading edge (Fig. 1a-e). Imaging of the Arp3 subunit through wide-field and TIRF microscopy revealed a homogeneous staining pattern along the P-domain and the leading edge of growth cones (Fig. $1 f, g$ ), without any enrichment in the C-domain. These results suggest that Arp2/3 is closely associated with the substrate at the leading edge, where we also found L1 to be enriched in the plasmalemma by TIRF microscopy (Fig. $1 h-j)$. Interestingly, Arp $2 / 3$ was shown to bind focal adhesion kinase (FAK) and vinculin and be recruited to nascent integrin adhesions to couple actin polymerization to adhesion formation (DeMali et al., 2002; Serrels et al., 2007). It is unknown if Arp2/3 is also recruited to L1 adhesions, but the colocalization of Arp2/3 with L1 makes it well suited to mediate actin polymerization at the leading edge and promote its protrusion.

\section{Arp2/3 influences leading edge protrusion, motility, and axon elongation}

Actin polymerization is essential for driving growth cone leading edge protrusion (Marsick et al., 2010; Gomez and Letourneau,
2013). Therefore, we sought to investigate the role of $\operatorname{Arp} 2 / 3$ during leading edge protrusion on L1 in response to NGF stimulation. NGF is an attractive guidance cue for DRG neurons during development and a potent stimulator of actin polymerization in vitro. We made time-lapse videos of DRG growth cones during NGF stimulation to analyze leading edge behavior with kymographs, either using $50 \mu \mathrm{M}$ CK666 to inhibit Arp2/3 or DMSO as a vehicle control (Nolen et al., 2009). We found that CK666 significantly reduced the average leading edge protrusion after NGF stimulation for $5 \mathrm{~min}$. (Fig. 2a-i). Yet, more revealing than the reduction in protrusion during Arp2/3 inhibition was the dynamic leading edge behavior. Shortly after initial NGF stimulation, the leading edge of CK666-treated growth cones would protrude but, arbitrarily, individual lamellipodial veils would collapse and retract toward the C-domain of the growth cone (Fig. 2g,h, yellow arrows). Yang et al. (2012) reported similar observations. At this point, the veil would remain collapsed or start protruding after a brief pause. A plausible explanation for veil collapse during Arp2/3 inhibition is the reduced number of actin branches at the leading edge, thus leaving a weakened actin network to abut against and resist the rearward membrane tension at individual veils. It has also been reported that retracting veils have a lower density of actin branches that protruding veils (Mongiu et al., 2007). Therefore, this experiment suggests that Arp2/3 is necessary for the protrusion and stability of the growth cone leading edge after NGF stimulation on L1.

Leading edge protrusion is associated with growth cone motility and axon elongation. Because Arp2/3 inhibition reduced leading edge protrusion on L1, we asked whether it would affect growth cone motility. We did time-lapse microscopy of growth cones treated either with DMSO or CK666. We found that CK666-treated growth cones had a 30\% reduction in their migration velocity over a $1 \mathrm{~h}$ period, compared with DMSO-treated growth cones (Table 1). Consistent with this result, overnight inhibition of Arp2/3 led to axon lengths that were $60-75 \%$ those of control axons, either with CK666 or by expression of the dominant-negative construct EGFP-CA (Table 1). This construct codes for the CA peptide, which has been shown to prevent Arp2/3 activation by WASP/Scar proteins (Hüfner et al., 2001; Strasser et al., 2004). These results suggest that Arp2/3 inhibition 
caused a motility deficit, which predictably led to shorter axons on L1.

Arp2/3, barbed ends, actin polymerization, and numbers of filopodia

Leading edge protrusion is driven by monomeric actin incorporation onto free F-actin barbed ends. We wanted to know what effect Arp2/3 inhibition would have on the formation and availability of free barbed ends in growth cones on L1. To this end, we used a barbed-end quantification assay, using DMSO or CK666 (Marsick et al., 2010; Marsick and Letourneau, 2011). Briefly, growth cones were pretreated with DMSO or CK666 for $4 \mathrm{~h}$, stimulated with NGF for $4 \mathrm{~min}$, permeabilized, bathed in a Rhodamine-actin solution, washed, fixed, and Rhodamineactin incorporation was quantified. We found that in DMSO-treated growth cones NGF stimulation led to a large increase in Rhodamine-actin incorporation at the leading edge, and that the Rhodamine-actin label would colocalize with Alexa 488 phalloidin staining (Fig. $2 j-n)$, thus, suggesting that the labeled actin had been incorporated into actin filaments. Similar results were published recently (Yang et al., 2012). On the other hand, NGF stimulation caused very little Rhodamine-actin incorporation into CK666treated growth cones (Fig. 2j,m,n). This experiment shows that most barbed ends created after NGF stimulation on L1 are located at the leading edge and are dependent on Arp2/3.

Given that Arp2/3 inhibition substantially reduced barbed-end numbers on L1, we investigated the effect it would have on total F-actin after NGF or Netrin stimulation. Netrin is a potent stimulator of actin polymerization and a chemoattractant (de la Torre et al., 1997; Marsick et al., 2010) in the guidance of RGC axons from the retina into the optic nerve head (Deiner et al., 1997). We inhibited Arp2/3 in DRG or retina explants with CK666 for $4 \mathrm{~h}$ before experiments or by overnight expression of the dominant-negative construct EGFP-CA. We applied global stimulation of NGF (DRG), Netrin (RGC), or media as a control to growth cones for 15 min, fixed, stained with Alexa 568 phalloidin, and quantified phalloidin fluorescent intensity. We found that in control conditions NGF or Netrin stimulation caused a large increase in the total F-actin content in growth cones (Fig. $3 a, b, e-g$ ). These growth cones also increased their surface area and acquired a lamellar morphology with an intense fringe of F-actin close to the leading edge (Fig. $3 a, b, h$ ). On the other hand, Arp2/3-inhibited growth cones had a small increase in F-actin content and surface area after NGF or Netrin treatment (Fig. $3 c-h$ ). Moreover, they


Figure 2. Arp2/3 mediates leading edge protrusion and barbed-end creation after global NGF stimulation on L1. $\boldsymbol{a}, \boldsymbol{b}, \boldsymbol{e}, \boldsymbol{f}, \mathrm{E} 7$ DRG explants were grown overnight on L1 and treated with DMSO or $50 \mu \mathrm{m}$ CK666 (Arp2/3 inhibitor) for $4 \mathrm{~h}$; growth cones were then imaged with phase contrast microscopy for $10 \mathrm{~min}$, before and after, global application of $50 \mathrm{ng} / \mathrm{ml}$ NGF. Representative kymographs depicting leading edge behavior during NGF stimulation (red bar) for ( $\boldsymbol{c}, \boldsymbol{d})$ DMSO-treated and $(\boldsymbol{g}, \boldsymbol{h})$ CK666-treated growth cones. Yellow arrows point to leading edge collapse. $i$, Quantification of leading edge protrusion $10 \mathrm{~min}$ after global NGF application. $\boldsymbol{k}-\boldsymbol{n}$, E7 DRG explants were grown on L1, treated with DMSO or CK666 for $4 \mathrm{~h}$, then with $50 \mathrm{ng} / \mathrm{ml} \mathrm{NGF}$ globally for 10 min, permeabilized with buffer containing phalloidin and Rhodamine-actin for 4 min, then fixed. $\boldsymbol{k}^{\prime} \boldsymbol{-} \boldsymbol{n}^{\prime}$, Rhodamine-actin (red) and phalloidin (green) labeling were overlaid to show barbed-end distribution in growth cones on L1. $\boldsymbol{j}$, Quantification of the average Rhodamine-actin incorporated into growth cones. Graphs show mean \pm SEM.

no longer had a lamellar morphology. These results further reinforce our findings that Arp $2 / 3$ is an important mediator of actin polymerization in growth cones on L1. Moreover, Arp2/3 activation promotes the protrusion and expansion of the leading edge.

Arp2/3 has been linked to filopodial formation in nonneuronal cells and growth cones (Machesky and Insall, 1998; Korobova and Svitkina, 2008). The mechanism was coined the 
Table 1. Arp2/3 role in axon outgrowth rate and length

\begin{tabular}{lll}
\hline & Laminin & L1 \\
\hline $\begin{array}{ll}\text { Axon outgrowth rate } \\
\quad\end{array}(\mu \mathrm{m} / \mathrm{h})$ & & \\
$\quad$ DMSO & $20.68 \pm 0.5881$ & $15.26 \pm 0.8684$ \\
$\quad$ CK666 & $18.02 \pm 0.8024$ & $10.76 \pm 0.5519$ \\
Axon length $^{b}(\mu \mathrm{m})$ & & \\
DMSO & $235.2 \pm 11.88$ & $198.2 \pm 11.10$ \\
CK666 & $195.6 \pm 12.61$ & $153.0 \pm 9.936$ \\
EGFP & $236.8 \pm 11.21$ & $239.5 \pm 13.39$ \\
EGFP-CA & $182.2 \pm 11.66$ & $144.1 \pm 12.01$ \\
\hline
\end{tabular}

${ }^{a}$ Measurements were done from time-lapse videos of E7 DRG explants' growth cones on L1 treated with DMSO or $50 \mathrm{M} \mathrm{CK666} 4 \mathrm{~h}$ prior to imaging. ${ }^{b}$ Measurements were done on dissociated low-density cultures on L1 from E7 DRG neurons grown for $24 \mathrm{~h}$, fixed, and stained with antibodies against neuronal $\beta 3$-tubulin. For pharmacological inhibition of Arp2/3 with CK666, neurons were allowed to adhere for $6 \mathrm{~h}$ before adding the drug.

convergent elongation model and proposes that actin filaments in filopodia can originate from the branched actin network that is dependent on Arp2/3 activity (Svitkina et al., 2003). We asked whether Arp2/3 influences numbers of filopodia in growth cones on L1. Thus, we counted filopodial numbers in DRG growth cones treated with DMSO or CK666, with and without NGF stimulation. We found that Arp2/3 inhibition reduced the extent to which NGF stimulation increased filopodial numbers in growth cones (Fig. 3i). This finding suggests that Arp 2/3 activity contributes to the stimulus-dependent increase in the number of filopodia in growth cones after NGF stimulation.

\section{Arp2/3 and actin retrograde flow}

Actin retrograde flow is controlled by three main factors: (1) myosin II activity pulling F-actin back from the leading edge (Lin et al., 1996; Medeiros et al., 2006); (2) elongating actin filaments that push on the membrane at the leading edge, resulting in increased membrane tension, which pushes back on the growing filaments, adding to the retrograde flow of the actin network; (Henson et al., 1999; Medeiros et al., 2006; Craig et al., 2012); and finally (3) linkage of actin filaments to substrate adhesions that restrict their backward flow relative to the substrate (Suter et al., 1998; Shimada et al., 2008; Marsick et al., 2012). Since Arp2/3 inhibition substantially reduced barbed-end numbers and total F-actin, we investigated what effect it would have on the retrograde flow of actin in growth cones on L1. To this end, we made kymographs from time-lapse videos of DRG and RGC growth cones transfected with mCherry-actin and EGFP-CA, or mCherry-actin and treated with DMSO or CK666 $4 \mathrm{~h}$ before imaging. We found that compared with controls, Arp2/3 inhibition led to a $15-30 \%$ reduction in the actin retrograde flow along veils, but no change in retrograde flow along filopodia (Fig. $4 a-$ $n)$. We hypothesize that the reduction in the actin retrograde flow after Arp $2 / 3$ inhibition is due to the reduced number of barbed ends capable of actin polymerization, which in turn generate less push against the leading edge membrane and the actin network toward the C-domain of the growth cone. We tested this idea using cytochalasin D to block monomeric actin incorporation into barbed ends and found a significant reduction in the actin retrograde flow rate, suggesting that monomeric actin incorporation into barbed ends can influence the actin retrograde flow (Fig. $4 m$ ). As a further control, we measured through immunostaining the activity of phospho-myosin light chain 2 (Ser19), the active form of the regulatory subunit for myosin II and a downstream target of ROCK, and found no difference in staining intensity between control and Arp2/3-inhibited growth cones (Fig. $4 o-r)$. However, application of the ROCK inhibitor, Y27632, did reduce staining for phospho-myosin light chain (Fig. 4q,r). This suggests that Arp2/3-dependent actin polymerization at the leading edge is a significant component in the driving force of the actin retrograde flow in growth cones.

\section{Arp2/3 role in guidance}

Chemoattraction depends on asymmetric leading edge protrusion on the side of the growth cone closest to the attractant source (Marsick et al., 2010; Dent et al., 2011). In turn, leading edge protrusion depends on the fine balance between actin polymerization and its retrograde flow at the leading edge. Since Arp $2 / 3$ inhibition caused a reduction in both leading edge protrusion and the actin retrograde flow, we asked which effect Arp2/3 inhibition would have during growth cone chemoattraction. NGF (DRG) or Netrin (RGC) was applied to one side of growth cones and their turning response measured. We found that Arp2/3 inhibition led to a $75-85 \%$ percent reduction in growth cone turning angle toward NGF or Netrin (Fig. $5 a-f$ ). Arp2/3-inhibited growth cones would generate protrusions toward the chemoattractant source, but often these protrusions would collapse, as occurred with the global NGF stimulation experiments (Figs. $\left.2 g, h, 5 d^{\prime}\right)$. This experiment shows that the actin nucleator Arp2/3 is required for optimal DRG and RGC growth cone chemotaxis toward NGF and Netrin, respectively, on L1.

This finding led us to ask whether localized activation of Arp2/3 on its own would be sufficient to trigger growth cone turning on L1. During chemotaxis, NGF and Netrin activate multiple signaling pathways that manipulate a variety of cellular processes in the growth cone (multiple ABPs, other cytoskeletal components, vesicular trafficking, calcium signals, etc.), all of which contribute to an optimal turning response. However, we wanted to isolate the effect of asymmetric Arp2/3 activation. We performed the same guidance assay mentioned above with modifications. We used the Chariot reagent to shuttle the VCA-GST peptide, which is capable of activating Arp2/3 (Hüfner et al., 2001; van der Gucht et al., 2005), into growth cones. We used this approach previously to deliver active ABPs into growth cones and study their effects during chemotaxis (Marsick et al., 2010). We applied Chariot + VCA-GST or Chariot $+\beta$-galactosidase as a control, and studied growth cone responses. We found that the Chariot + VCA-GST combination was capable of steering growth cones toward its source at angles significantly larger than the Chariot $+\beta$-galactosidase combination (Fig. $5 g$ ). More importantly, turning toward the Chariot + VCA-GST source was abolished in the presence of the Arp2/3 inhibitor CK666, indicating that Arp2/3 mediates the turning response elicited by Chariot + VCA-GST. Regarding the smaller turning angles triggered by Chariot + VCA-GST compared with NGF or Netrin, it is reasonable to suggest they are the result of the missing parallel signaling pathways that are normally activated by these chemoattractants, in addition toArp2/3-mediated actin polymerization. These results establish that asymmetrical activation of the Arp $2 / 3$ complex is sufficient to trigger growth cone turning on L1.

We determined that Arp $2 / 3$ is required to follow a soluble chemoattractant gradient on L1. Unable to establish a soluble chemorepellent assay that would consistently repel growth cones away from the source, we opted for a membrane-bound chemorepellent assay with alternating stripes of substrate-bound L1 and L1 + ephrin A2. E7 temporal retina stripes were laid perpendicular to the stripes and allowed to grow for $40 \mathrm{~h}$; the final $24 \mathrm{~h}$ were under the effect of DMSO or CK666 (Fig. 5h). This approach allowed explants to initiate and grow neurites before Arp2/3 inhibition, thus, eliminating any possible defect that Arp $2 / 3$ inhibition might cause during neuritogenesis, and allow- 

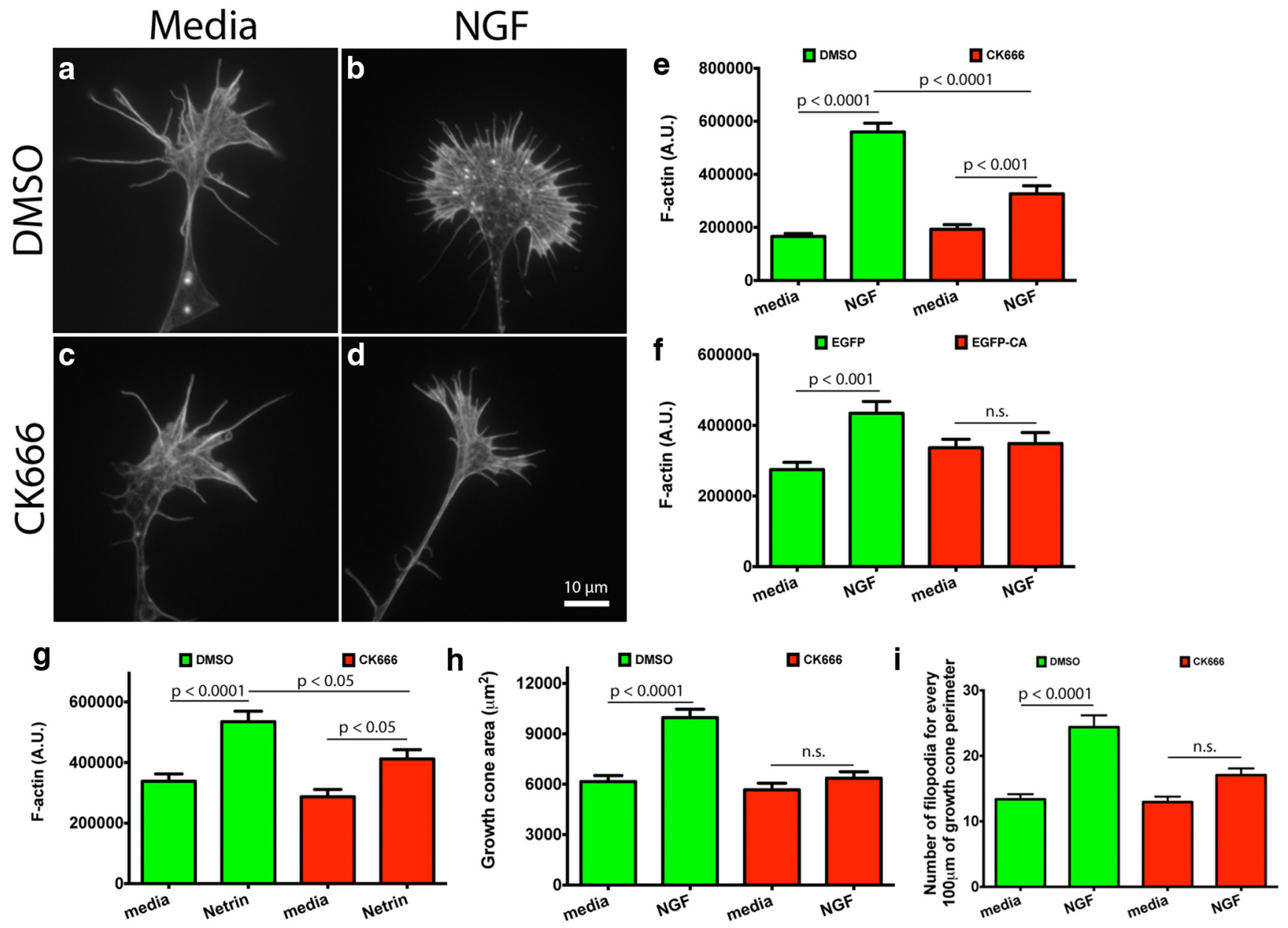

Figure 3. Arp2/3 mediates actin polymerization, surface area expansion, and increase in filopodial numbers in growth cones on L1. $\boldsymbol{a}-\boldsymbol{d}$, E7 DRG explants were grown overnight on L1, treated with DMSO or $50 \mu \mathrm{m}$ CK666 for $4 \mathrm{~h}$, stimulated with $50 \mathrm{ng} / \mathrm{ml}$ NGF or control media for $15 \mathrm{~min}$, fixed, stained with Alexa Fluor 488 phalloidin, (e) and its fluorescent intensity quantified. $\boldsymbol{f}$, Quantification of Alexa Fluor 488 phalloidin fluorescent intensity bound to DRG growth cones transfected with EGFP, or the EGFP-CA (Arp2/3 dominant-negative construct). $\boldsymbol{g}$, Quantification of Alexa Fluor 488 phalloidin fluorescent intensity bound to RGC growth cones treated with DMSO or $50 \mu \mathrm{M} \mathrm{CK666} \mathrm{for} 4 \mathrm{~h}$, stimulated with $500 \mathrm{ng} / \mathrm{ml}$ Netrin or media as a control for 15 min, fixed, and stained with phalloidin. $\boldsymbol{h}$, Measured DRG growth cone area. $\boldsymbol{i}$, Average number of filopodia for every $100 \mu \mathrm{m}$ of growth cone perimeter. Graphs show mean \pm SEM.

ing us to study guidance only. Explants were fixed, and axon trajectories were revealed by immunolabeling with anti- $\beta 3$ tubulin, while staining with anti-ephrin A2 assessed the integrity of the ephrin A2 lanes. We found that explants treated with DMSO had axons that consistently remained within the L1 lanes, thus avoiding the $\mathrm{L} 1+$ ephrin A2 lanes (Fig. 5i-k). Interestingly, treatment with CK666 led to a range of guidance deficits that ranged from (1) growth cones ignoring ephrin A2 and crossing the boundaries, (2) continued migration over the ephrin A2-containing lanes, and (3) axonal branching over the ephrin A2-containing lanes (Fig. 5l-p). These results suggest that Arp2/ 3 -dependent actin polymerization is necessary for an effective avoidance response to the membrane-bound chemorepulsive cue ephrin A2 on L1.

\section{Laminin results}

Laminin as a substrate

In culture, L1 promotes the formation of broad lamellar growth cones. We showed that Arp2/3 inhibition on this substrate causes growth cones to have deficiencies in cytoskeletal dynamics and guidance. In view of these results, we asked whether such deficiencies would also arise on a substrate where growth cones are more compact and filopodial, and perhaps less dependent on the branched actin network organized by Arp $2 / 3$. For this reason we decided to use laminin, which is an ECM protein found in most organs and tissues and has also been shown to be an endogenous substrate for growing axons (McLoon et al., 1988).

Arp2/3 distribution in growth cones on laminin

To assess Arp2/3 function in growth cones on laminin, we first asked where it was localized. Through immunostaining, we found Arp2/3 subunit Arp3 to be localized homogeneously across the growth cone $\mathrm{P}$-domain, without any enrichment at the leading edge (Fig. 6a-d). However, Mongiu et al. (2007) reported that chick DRG growth cones do have Arp2/3 subunits enriched at their veils through electron microscopy. This discrepancy across labs is most likely due to the higher resolution and sensitivity of their screening methods. The fact that we were able to detect Arp2/3 subunit enrichment at the leading edge in growth cones on L1 through immunostaining might reflect a denser branched actin network on this substrate, than on laminin.

Arp2/3 motility and axon length

We showed that $\operatorname{Arp} 2 / 3$ was required for optimal growth cone motility on L1. Therefore, we asked whether Arp2/3 was also required for motility on laminin. We made time-lapse videos of 

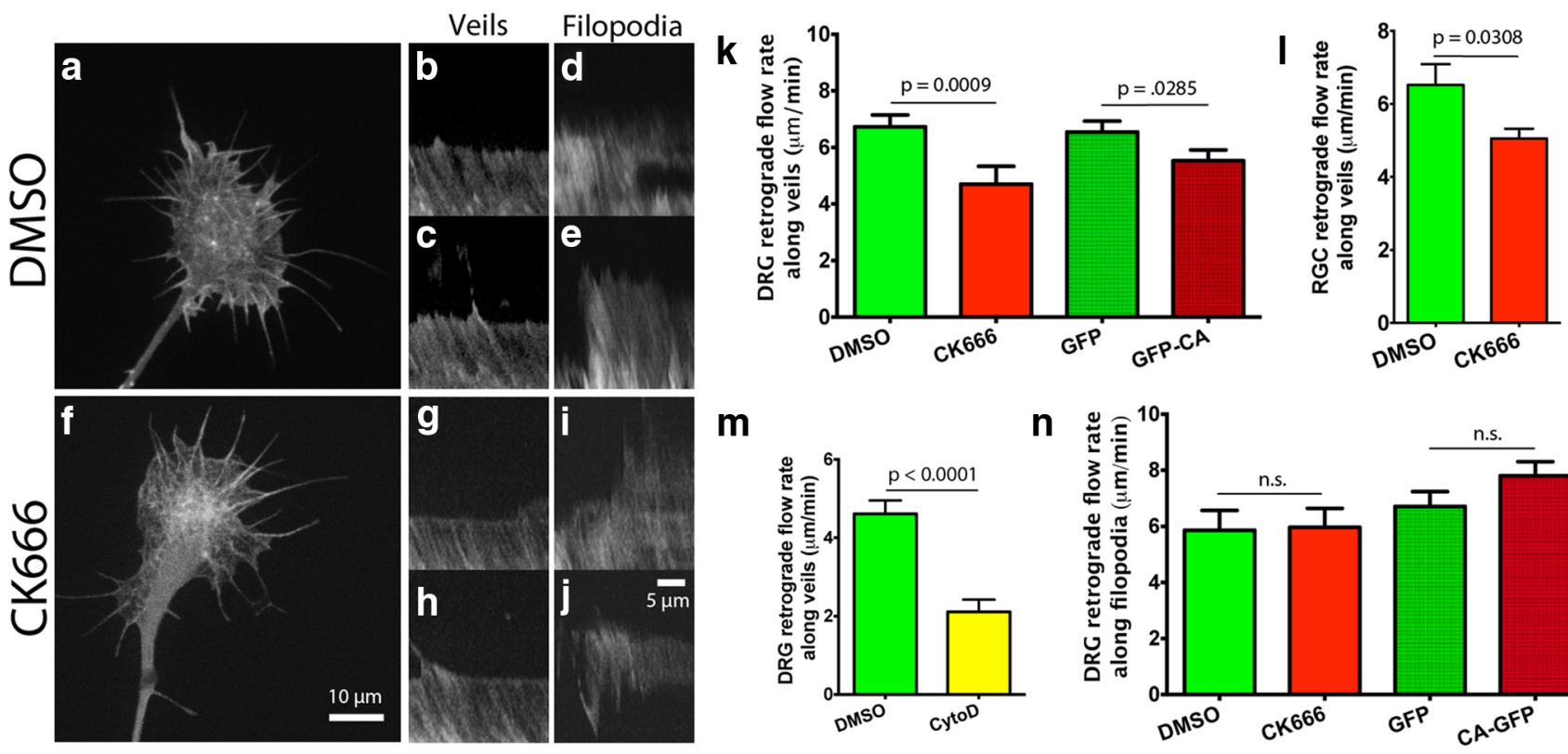

m

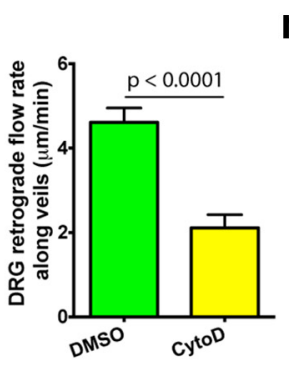

n

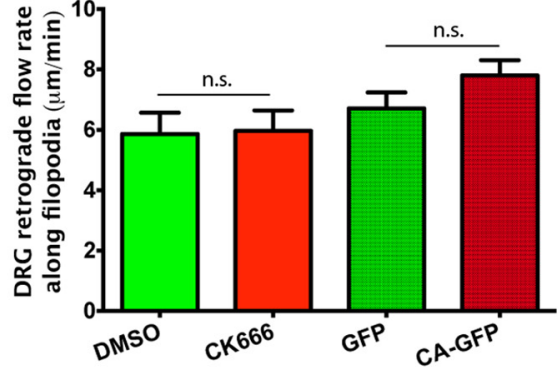

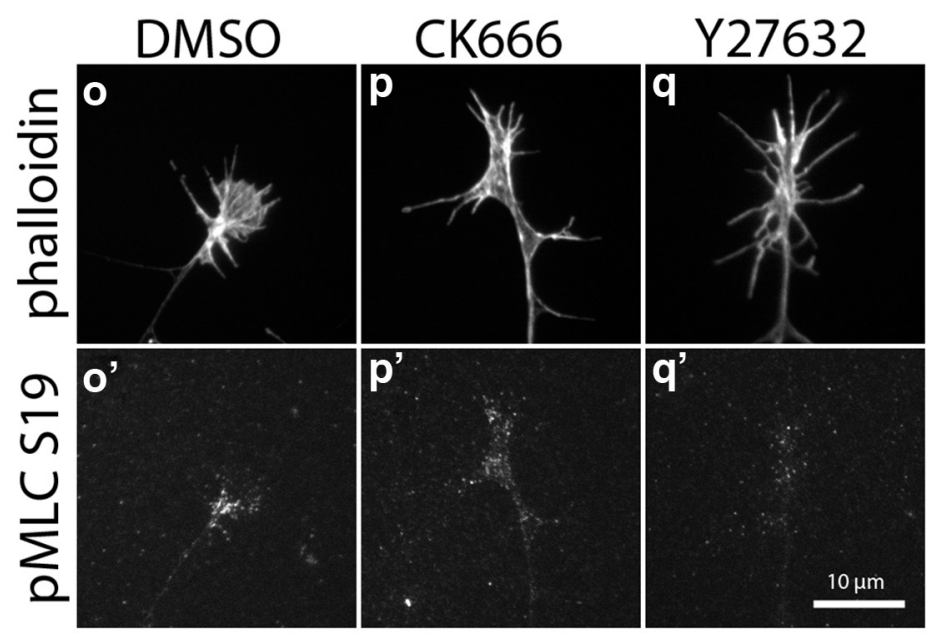

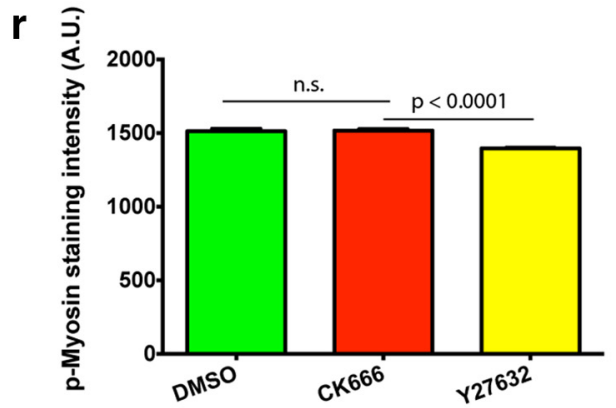

Figure 4. Arp2/3 inhibition reduces actin retrograde flow at the leading edge of growth cones on $\mathrm{L} 1$, independent of myosin II activity. $\boldsymbol{a}, \boldsymbol{f}$, E7 DRG or RGC neurons were transfected with $\mathrm{mCherry-actin} \mathrm{and} \mathrm{grown} \mathrm{overnight} \mathrm{on} \mathrm{L1.} \mathrm{Arp2/3} \mathrm{was} \mathrm{inhibited} \mathrm{by} \mathrm{coexpression} \mathrm{of} \mathrm{EGFP-CA} \mathrm{with} \mathrm{mCherry-actin,} \mathrm{or} \mathrm{by} 50 \mu \mathrm{m}$ CK666 treatment $4 \mathrm{~h}$ before imaging. Growth cones were imaged in a spinning disc confocal microscope and kymographs made from these time-lapse videos. $\boldsymbol{b}, \boldsymbol{c}, \boldsymbol{g}, \boldsymbol{h}$, Representative kymographs along veils and $(\boldsymbol{d}, \boldsymbol{e}, \boldsymbol{i}, \boldsymbol{j})$ filopodia of DMSO-and CK666-treated growth cones. $\boldsymbol{k}, \boldsymbol{I}$, Quantification of the actin retrograde flow along veils of DRG and RGC growth cones and $(\boldsymbol{m})$ the effect that $2 \mu \mathrm{m}$ cytochalasin D has on it. Note that in this experiment DMSO and cytochalasin D were added 3 min before imaging, instead of the normal $4 \mathrm{~h}$, hence the different DMSO control rates. $n$, Quantification of the actin retrograde flow along filopodia of DRG growth cones. $\mathbf{0}-\boldsymbol{q}^{\prime}$, As a control, DRG growth cones on L1 were treated with DMS0, $50 \mu \mathrm{M} \mathrm{CK666,} \mathrm{or} 10 \mu \mathrm{m}$ Y27632 for $4 \mathrm{~h}$, fixed, and stained with phalloidin and an antibody against phospho myosin light chain (Ser19), (r) and anti-pMLC average fluorescent intensity quantified. Graphs show mean \pm SEM.

DRG explants with and without Arp2/3 inhibition. As before, explants were imaged for $1 \mathrm{~h}$ and the velocity of individual growth cones measured. We found that Arp2/3 inhibition did not cause a significant deficit in migration velocity, compared with control growth cones (Table 1). This suggests that Arp2/3 is not crucial for effective growth cone motility on laminin, although it is on L1.

Even though growth cone motility on laminin over a $1 \mathrm{~h}$ period was not significantly affected by Arp $2 / 3$ inhibition, we asked whether the prolonged effect of overnight inhibition would reduce axon elongation on laminin, as it did on L1. For this purpose, we made low-density cultures of DRG neurons that were either transfected with EGFP-CA or treated with CK666 $5 \mathrm{~h}$ after plating to inhibit Arp2/3, grown overnight, fixed, and stained with anti- $\beta 3$ tubulin. We found that Arp $2 / 3$ inhibition, by either of these two methods, reduced average axon length to $80 \%$ that of controls (Table 1). This shows that prolonged inhibition of Arp2/3 function will reduce axon length on laminin, although not as severely as it did on L1.

\section{Arp2/3 effect on actin polymerization and filopodia}

Arp2/3 inhibition led to a drastic reduction in the increase of F-actin on L1 after NGF stimulation. Consequently, we asked whether Arp2/3 inhibition would have a similar effect on laminin, where growth cones are compact and filopodial. We pretreated DRG explants with DMSO or CK666 for $4 \mathrm{~h}$, followed by global NGF stimulation, or media as a control, for $15 \mathrm{~min}$. Growth cones were then fixed, stained with Alexa 488 phalloidin, and imaged. We found NGF stimulation led to a large increase in total F-actin and growth cone surface area, whereas Arp2/3inhibited growth cones responded with only a minor increase in F-actin and area after NGF treatment (Fig. $6 e-j$ ). Not only was 

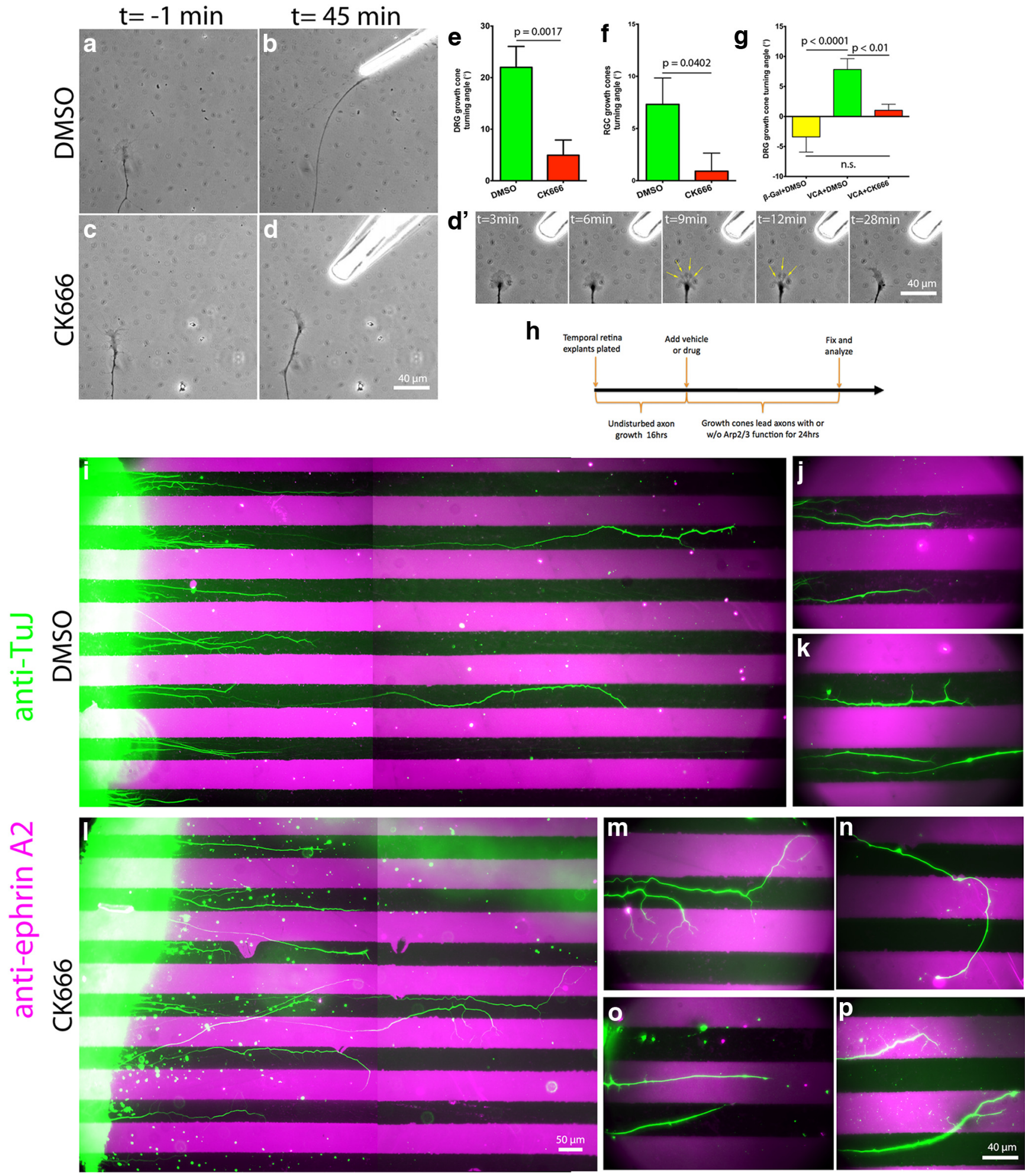

Figure 5. Arp2/3 activity is necessary for efficient guidance and sufficient to trigger growth cone turning on L1. E7 DRG or RGC explants were grown on L1 overnight and treated with DMSO or $50 \mu \mathrm{m}$ CK666 $4 \mathrm{~h}$ before the turning assay. NGF (DRG) or Netrin (RGC) gradients were established with a micropipette located at $45^{\circ}$ and $\sim 80 \mu \mathrm{m}$ from the growth cone leading edge. $\boldsymbol{a}-\boldsymbol{d}$, Representative images of DRG growth cones $1 \mathrm{~min}$ before and $45 \mathrm{~min}$ after positioning the micropipette. $\boldsymbol{d}^{\prime}$, Montage of Arp2/3-inhibited growth cone response to the NGF gradient showing multiple points of veil collapse (yellow arrows). $\boldsymbol{e}, \boldsymbol{f}, \mathrm{DRG}$ and RGC growth cone turning angle quantification. $\boldsymbol{g}$, Turning angle quantification for DRG growth cones on L1 in response to a gradient of Chariot complexed to VCA-GST or $\beta$-galactosidase as a control, delivered as above. $\boldsymbol{h}$, Diagram depicting the experimental design for the substrate-bound ephrin A2 chemorepulsive assay. $\boldsymbol{i}, \boldsymbol{I}$, Boundary assay for control (DMSO) and Arp2/3-inhibited (CK666) retina explants (left) with alternating lanes of laminin (black) and ephrin A2 + laminin (magenta) with axons stained with antibodies against the neuronal $\beta 3$-tubulin (green). $\boldsymbol{j}$, $\boldsymbol{k}$, Distal tips of control and ( $\boldsymbol{m}-\boldsymbol{p}$ ) Arp2/3-inhibited axons shown at higher magnification. Note the guidance deficits and the multiple processes sprouted by Arp2/3-inhibited axons over the ephrin A2 lanes. Graphs show mean \pm SEM. 

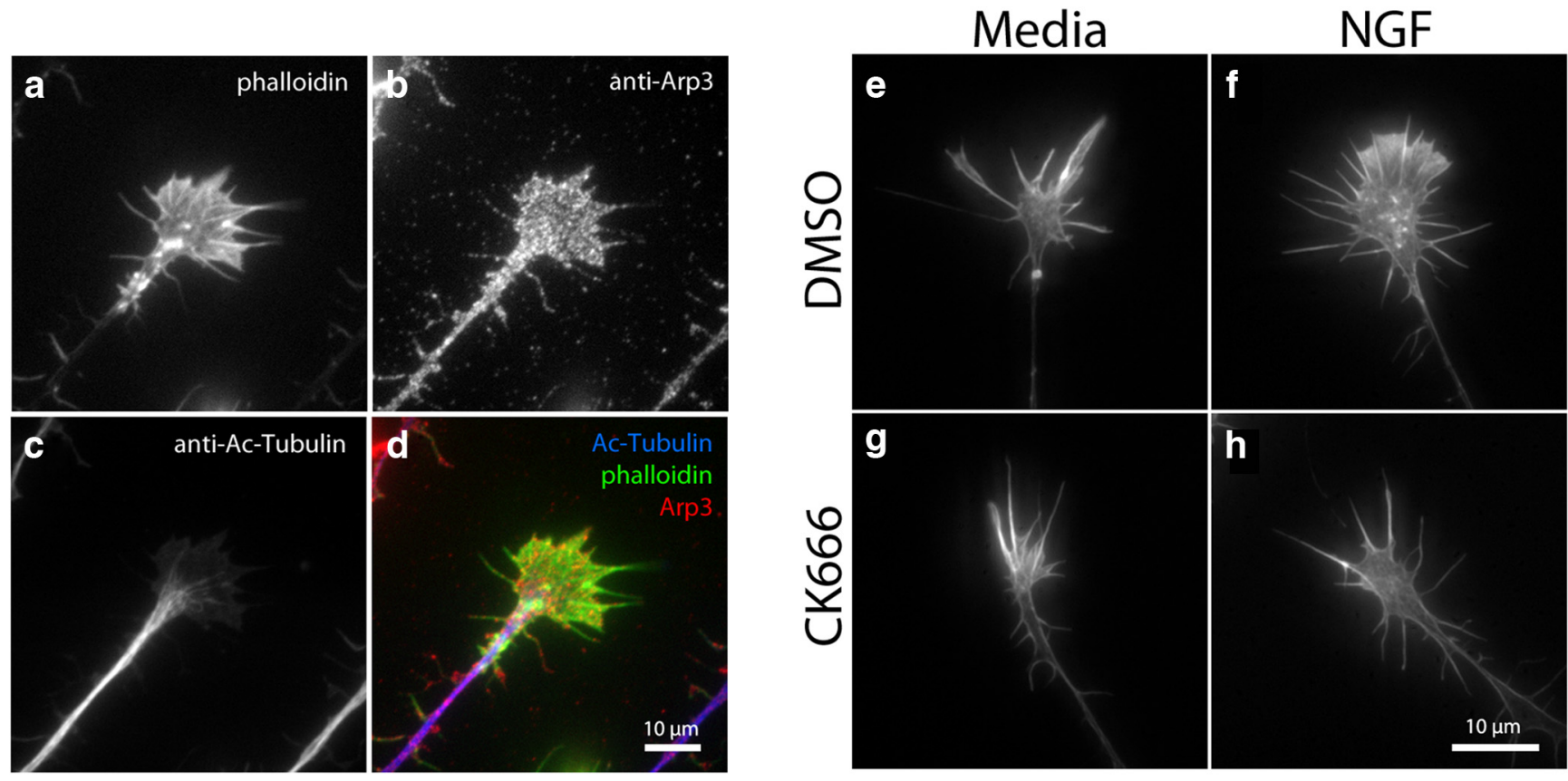

i
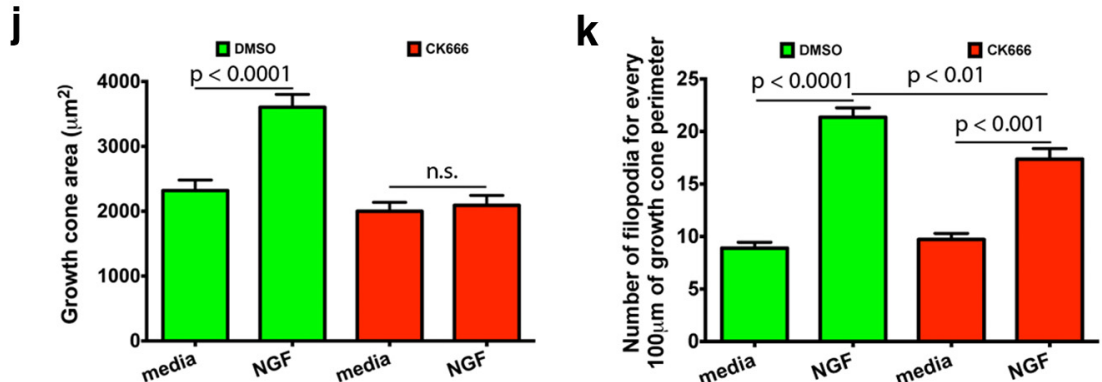

Figure 6. Arp2/3 mediates actin polymerization, surface area expansion, and increase in number of filopodia in growth cones after NGF stimulation on laminin. $\boldsymbol{a}-\boldsymbol{d}$, E7 DRG growth cones on laminin, treated with NGF, and stained with phalloidin and antibodies against Arp3 and acetylated $\alpha$-tubulin (Ac-Tubulin). $\boldsymbol{e}-\boldsymbol{h}$, E7 DRG growth cones on laminin treated with DMS0 or CK666 for $4 \mathrm{~h}$, stimulated with NGF or media as control, fixed, stained with Alexa Fluor 488 phalloidin, (i) and phalloidin fluorescent intensity quantified. $\boldsymbol{j}$, Quantification of growth cone area. $\boldsymbol{k}$, Average number of filopodia for every $100 \mu \mathrm{m}$ of growth cone perimeter. Graphs show mean \pm SEM.

there a quantitative difference in growth cone F-actin, but a qualitative difference as well. Arp2/3-inhibited growth cones had fewer veils than controls, consistent with Arp2/3 role in nucleating a branched actin network. This result shows that Arp2/3 mediates actin polymerization in growth cones on laminin after NGF stimulation, as it does on L1.

Similar to other groups using non-neuronal and neuronal cells (Machesky and Insall, 1998; Svitkina et al., 2003; Korobova and Svitkina, 2008), we found that Arp2/3 inhibition reduced the number of NGF-induced filopodia in growth cones on L1. Therefore, we asked whether Arp2/3 inhibition would have any effect on the number of NGF-induced filopodia in growth cones on laminin. We found that Arp2/3 inhibition led to a reduction in the number of filopodia in growth cones after NGF stimulation, like on L1 (Fig. 6k). These results suggest that Arp2/3 is involved in the formation of filopodia on laminin and L1.

Arp2/3 and the retrograde flow of actin

Arp2/3 inhibition led to a reduction in the rate of the actin retrograde flow along growth cone veils on L1. Presumably, this decreased rate was due to a reduced retrograde push exerted on the actin network, because of the reduced force exerted by actin polymerization against the leading edge membrane. Since Arp2/3 inhibition reduced actin polymerization in growth cones on laminin, we asked which effect Arp2/3 inhibition would have on the actin retrograde flow on laminin. For this purpose, we did time-lapse videos of DRG neurons transfected with mCherryactin and grown overnight. To our surprise, Arp2/3 inhibition led to an increased actin retrograde flow rate along growth cone veils but not along filopodia on laminin, regardless of whether CK666 or EGFP-CA was used to inhibit Arp2/3 (Fig. 7a-h). Recently, Yang et al. (2012) reported similar results in Aplysia neuronal growth cones and concluded that the actin network assembled by Arp2/3 restricts the myosin II-dependent contractility of the actin network in a ROCK-independent manner (Yang et al., 2012). When we measured phospho-myosin light chain 2 (Ser19), a regulatory subunit for myosin II and downstream target of ROCK, similar to our results on L1 and Yang et al. (2012), we found no difference in the myosin II activity after Arp2/3 inhibition (Fig. 7i-o). As a control, the ROCK inhibitor Y27632 did reduce staining for phosphomyosin light chain 2 (Fig. $7 k, l$ ). In addition, we found that the actin retrograde flow along filopodia was indistinguishable between control and Arp2/3-inhibited growth cones (Fig. 6h) further suggesting that the increased rate in the actin retrograde flow along veils is not a result of increased myosin II activity.

Because it is unlikely that Arp2/3 inhibition would increase the retrograde flow of actin by promoting actin polymerization, 
we examined integrin-based adhesions in growth cones on laminin, since the adhesion-based clutch is a determinant of the actin retrograde flow. Recent reports in non-neuronal cells have shown that Arp2/3 is recruited to nascent integrinbased adhesions and interacts with vinculin and FAK, as a way to couple actin polymerization to newly formed adhesions (DeMali et al., 2002; Serrels et al., 2007). We transfected DRG neurons with paxillin-GFP, a marker of integrin adhesions, and mCherry-CA, grew them overnight, and imaged cell-substrate interactions by TIRF microscopy. Consistent with the increased actin retrograde flow rate after Arp2/3 inhibition, we found that the mean area of paxillin-GFP puncta was $70 \%$ that of controls (Fig. $8 a-$ e). Moreover, these growth cones also had a reduced number of paxillin-GFP puncta compared with controls (Fig. $8 f$ ). It is likely that the smaller size and reduced number of adhesions are not as effective at engaging the overlying flow of actin filaments, and as a result the actin retrograde flow rate is faster. A recent report on fibroblasts showed that adhesion size was not affected by Arp2/3 inhibition, only their orientation relative to the leading edge (Wu et al., 2012). Our experiments suggest that on laminin the size and number of integrin-based adhesions in growth cones is influenced by Arp2/3 activity.

\section{Arp2/3 and guidance}

It is thought that local stimulation of actin polymerization steers the growth cone leading edge toward attractive guidance cues (Marsick et al., 2010; Dent et al., 2011). We know that Arp $2 / 3$ inhibition reduced the NGF-stimulated increase in F-actin content in growth cones on laminin. Arp2/3 inhibition also reduced actin polymerization in DRG and RGC growth cones on L1, which was associated with a guidance deficit toward the chemoattractants NGF and Netrin, respectively. Therefore, we asked whether the reduction in actin polymerization on laminin after Arp2/3 inhibition would also affect growth cone chemoattraction toward NGF. As before, we applied NGF to one side of growth cones and measured their turning responses. Surprisingly, we found that Arp2/3 inhibition did not reduce chemoattraction toward NGF, but instead we observed an increase in the turning angles (Fig. 9a-e). This experiment suggests that Arp2/3-mediated actin polymerization is not required for chemoattractive turning on laminin, as opposed to on L1.

Because Arp2/3 inhibition did not cause deficient attractive guidance toward NGF on laminin, we asked whether its unilateral
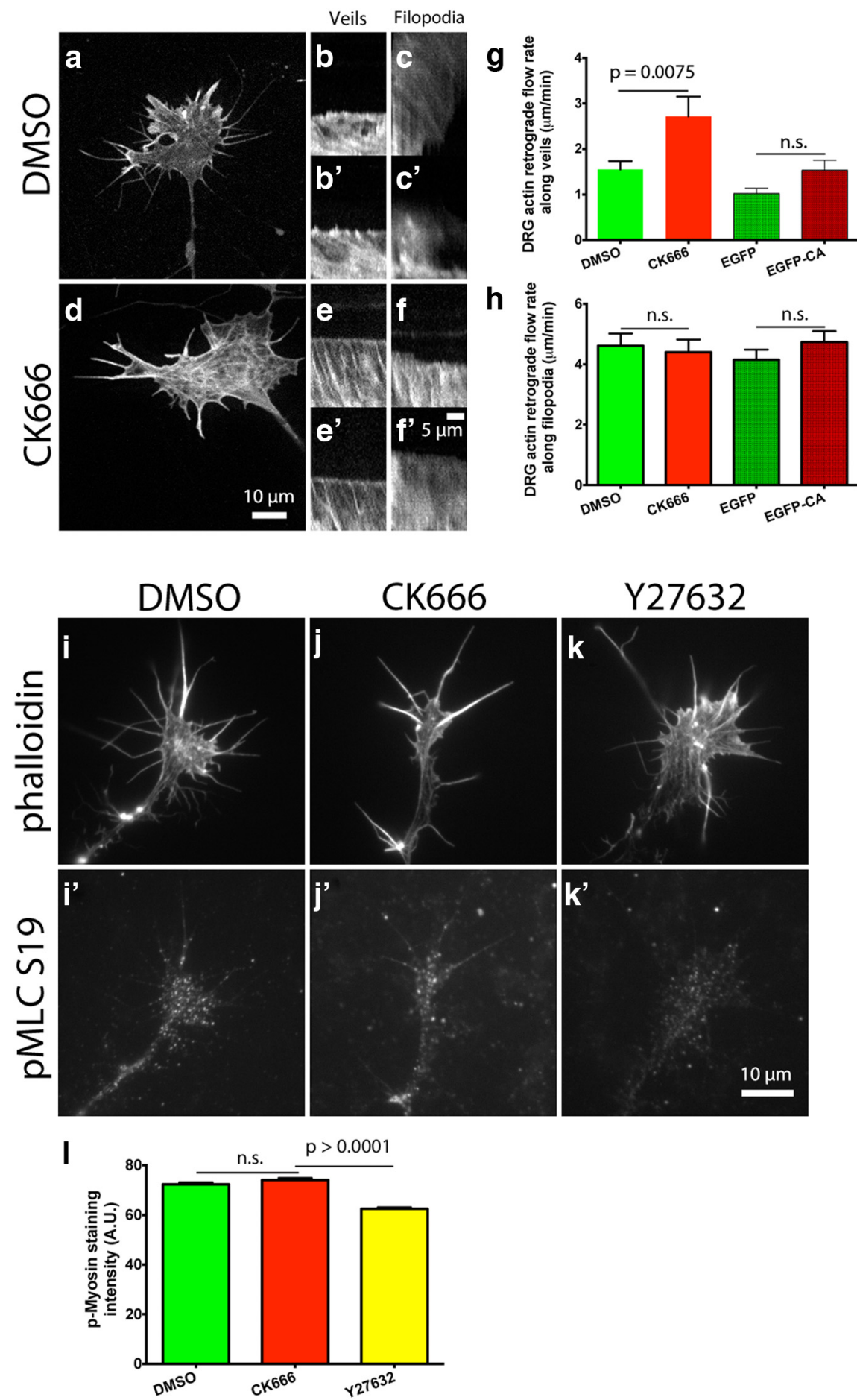

Figure 7. Arp2/3 inhibition increases the actin retrograde flow rate at the leading edge of growth cones on laminin in a myosin II-independent manner. $\boldsymbol{a}, \boldsymbol{d}$, E7 DRG neurons were transfected with mCherry-actin and grown overnight on laminin. Arp2/3 was inhibited by coexpression of EGFP-CA with mCherry-actin, or by $50 \mu \mathrm{m}$ CK666 treatment $4 \mathrm{~h}$ before imaging. Growth cones were imaged in a spinning disc confocal and kymographs made from these time-lapse videos. $\boldsymbol{b}, \boldsymbol{b}^{\prime}, \boldsymbol{e}, \boldsymbol{e}^{\prime}$, Representative kymographs along veils and $\left(\boldsymbol{c}, \boldsymbol{c}^{\prime}, \boldsymbol{f}, \boldsymbol{f}^{\prime}\right)$ filopodia of DMSO- and CK666-treated growth cones. $\boldsymbol{g}, \boldsymbol{h}$, Quantification of the actin retrograde flow rate along veils and filopodia. $\boldsymbol{i}-\boldsymbol{k}^{\prime}$, As a control, DRG growth cones on L1 were treated with DMSO, $50 \mu \mathrm{M} C K 666$, or $10 \mu \mathrm{M} \mathrm{Y27632}$ for $4 \mathrm{~h}$, fixed, and stained with phalloidin and an antibody against phospho myosin light chain (Ser19), (I) and anti-pMLC average fluorescent intensity quantified. Graphs show mean \pm SEM. 

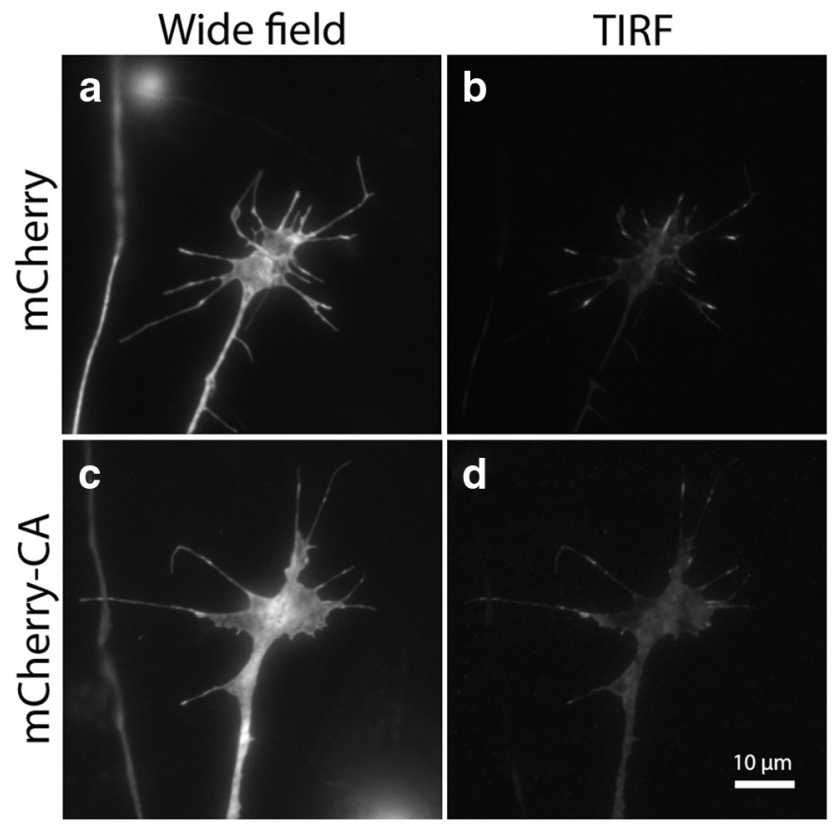

(
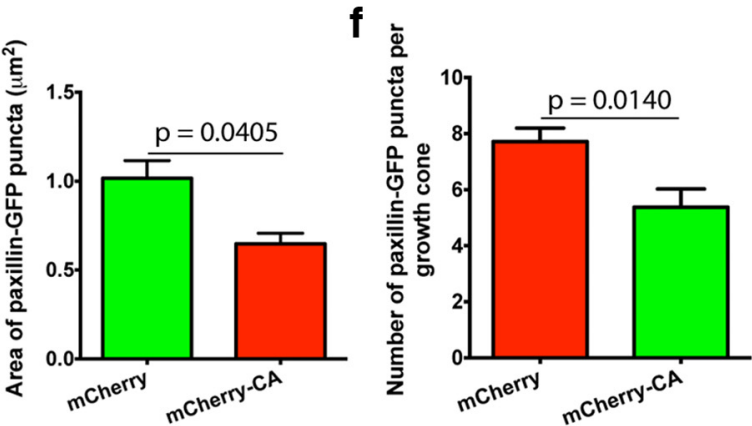

Figure 8. Arp2/3 inhibition reduces the area and number of paxillin-GFP puncta in growth cones on laminin. $\boldsymbol{a}-\boldsymbol{d}$, E7 DRG neurons were transfected with paxillin-GFP and mCherry-CA, grown overnight on laminin, and imaged through wide-field and TIRF microscopy.e, $\boldsymbol{f}$, Thresholded TIRF images were used to measure the area and number of paxillin-GFP puncta.

we used a micropipette to deliver a gradient of Chariot + VCAGST or Chariot + B-galactosidase, as control. We found that the Chariot + VCA-GST peptide complex could not elicit growth cone turning on laminin toward the gradient source (Fig. $9 f$ ). This indicates that localized activation of Arp $2 / 3$ is insufficient to trigger growth cone turning on laminin, as opposed to on an L1 substrate, and suggests that other cellular processes are required to execute attractive chemotactic turning on laminin.

Since Arp2/3 inhibition did not reduce chemoattraction toward NGF, we asked whether Arp2/3 was also dispensable for contact-mediated chemorepulsion. When we performed this experiment on L1, we found that RGC axons exhibited a variety of guidance deficits. We made a boundary assay with alternating stripes of laminin or laminin + ephrin A2. E7 temporal retina stripes were laid perpendicular to the stripes and allowed to grow for $40 \mathrm{~h}$, the final $24 \mathrm{~h}$ under the effect of DMSO or CK666. Explants were fixed, and axon trajectories were revealed with anti- $\beta 3$ tubulin, while the integrity of the ephrin A2 lanes was indicated with anti-ephrin A2. Despite being shorter, Arp2/3inhibited axons were just as responsive to the contact-mediated chemorepulsive effects of the ephrin A2-containing lanes as the control group (Fig. $9 g-l$ ). This result suggests that Arp2/3 function is not required for efficient contact-mediated chemorepulsion on laminin, as it is on L1.

\section{Discussion}

Here we investigated the role of Arp2/3 in growth cone leading edge protrusion, actin dynamics, and guidance. Furthermore, we examined how the substrate influenced these processes. We found that Arp2/3 inhibition reduced F-actin content, number of filopodia, and axon length in DRG neurons, regardless of the substrate. However, we found that Arp2/3 inhibition had substrate-dependent effects on actin retrograde flow and growth cone guidance.

Similar to most reports on non-neuronal cells, we found Arp2/3 was necessary for NGF-induced growth cone leading edge protrusion on L1 (Fig. 2a-h). Interestingly, Arp2/3-inhibited veils would protrude in response to NGF but then collapse (Figs. $\left.2 g, h, 5 d^{\prime}\right)$, perhaps due to the reduced branch density in the actin network at the leading edge. It is possible that the centrifugal leading edge expansion induced by actin polymerization causes an increase in centripetal membrane tension that compresses the Arp2/3-inhibited actin network underneath it, leading to veil collapse (Craig et al., 2012). This compressive tension can explain the actin filaments oriented parallel to the leading edge during Arp2/3 inhibition, as described by Strasser et al. (2004) (Fig. 4c). These results suggest that Arp2/3 is integral for the protrusion and stability of the leading edge on L1.

Consistent with an Arp2/3 role in leading edge protrusion, immunostaining revealed enrichment of subunits p34 and Arp3 at the leading edge (Fig. $1 a-g$ ). Moreover, in the barbed-end assay most Rhodamine-actin incorporation occurred at the leading edge, colocalized with the distal most phalloidin staining of F-actin, and was dependent on Arp2/3 function (Fig. $2 j-n^{\prime}$ ). Thus, Arp2/3-mediated actin polymerization promotes leading edge protrusion in growth cones on L1.

Arp2/3 inhibition reduced actin polymerization in growth cones regardless of the substrate, albeit incompletely (Figs. $3 a-g$, $6 e-i)$. This suggests either (1) incomplete inhibition of Arp2/3 and/or (2) there is also activation of other ABPs by NGF to promote barbed-end creation and F-actin polymerization. Potential candidates are other actin nucleators like the formins, Ena/VASP proteins, and the F-actin severing protein $\mathrm{ADF} /$ cofilin (Marsick et al., 2010; Dent et al., 2011). Overall, this suggests that Arp2/3 is an important mediator of actin polymerization in growth cones on L1 and laminin.

In accordance with the convergent elongation model (Svitkina et al., 2003), Arp2/3 inhibition reduced the number of filopodia in growth cones on both substrates, but only during NGF stimulation (Figs. 3i, 6k). These results suggest that actin branches created by Arp $2 / 3$ after NGF stimulation mediate filopodial formation, regardless of growth cone substrate.

Interestingly, inhibition of the actin nucleator Arp2/3 led to differential changes in the rate of the actin retrograde flow. On L1, Arp2/3 inhibition reduced the rate of the actin retrograde flow along veils by $15-30 \%$, which is likely due to the reduced number of barbed ends polymerizing actin at the leading edge. The idea is that the actin retrograde flow is influenced by the "pull" exerted by myosin II contractile activity along actin filaments, and by a "push back" from membrane tension, as polymerizing actin filaments expand the actin network and push against the leading edge (Henson et al., 1999; Medeiros et al., 2006; Craig et al., 2012). This model is supported by our experiments with cytochalasin D (Fig. $4 a-m$ ), whereby inhibition of actin polymerization significantly reduced its retrograde flow. These results suggest that Arp2/3-dependent actin polymerization at the leading edge influences the rate of the actin retrograde flow. 
DMSO

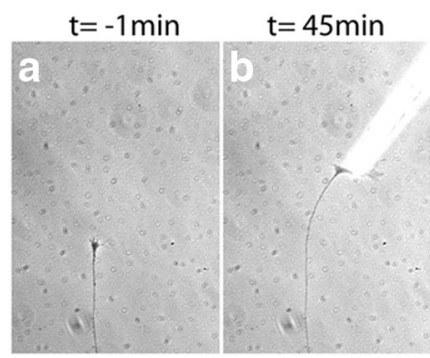

CK666

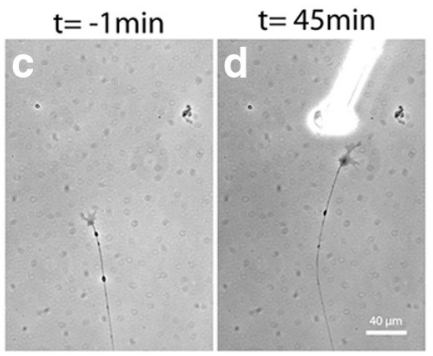

e

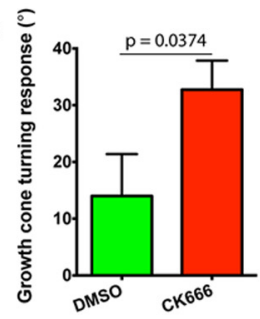

f

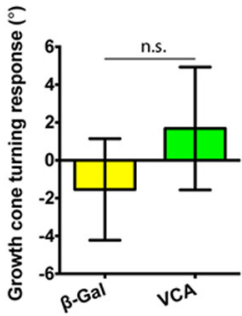

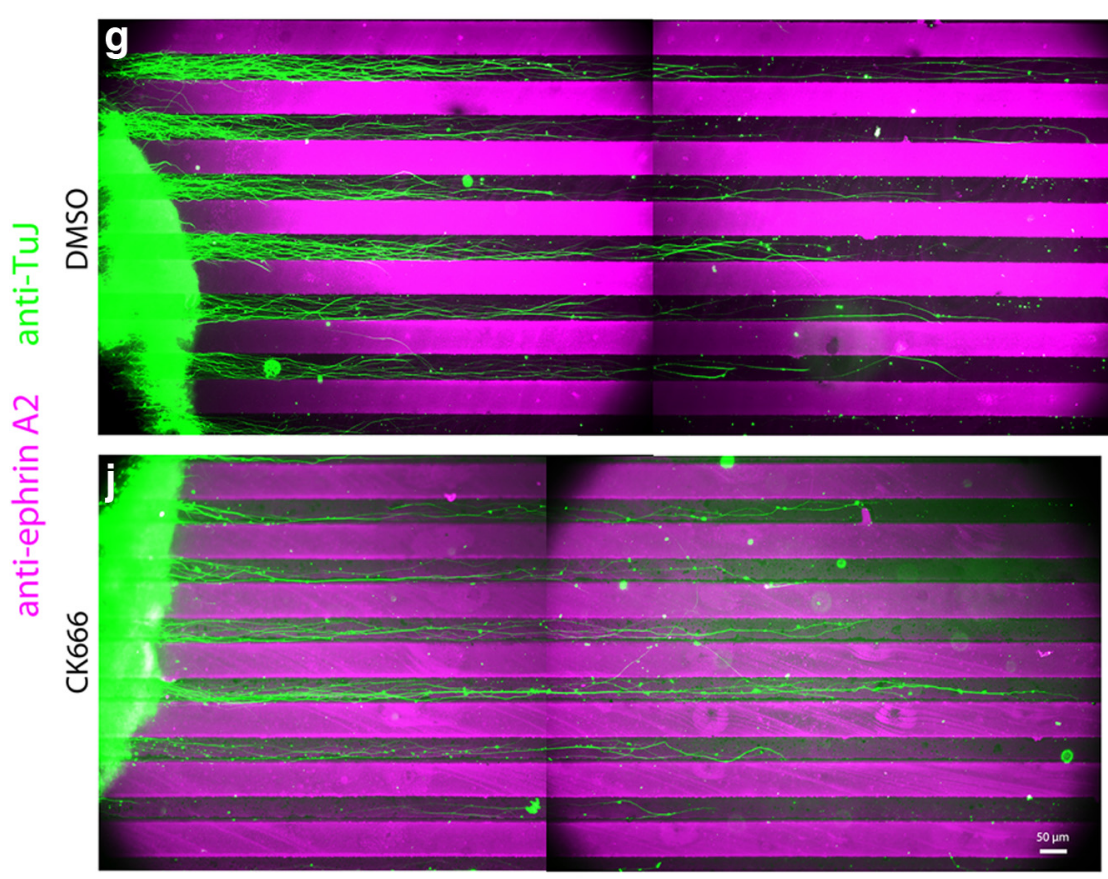
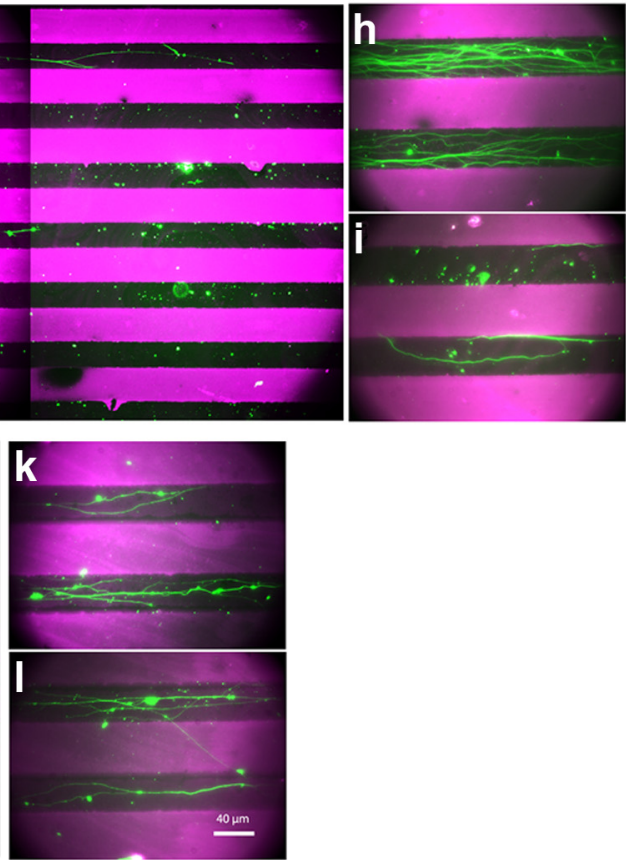

Figure 9. Arp2/3 activity is not necessary for efficient guidance and insufficient to trigger growth cone turning on laminin. E7 DRG explants were grown on laminin and treated with DMSO or 50 $\mu \mathrm{M}$ CK666 $4 \mathrm{~h}$ before the turning assay. An NGF gradient was established with a micropipette located at $45^{\circ}$ and $\sim 80 \mu \mathrm{m}$ from the growth cone leading edge. $\boldsymbol{a}-\boldsymbol{d}$, Representative images of DRG growth cones $1 \mathrm{~min}$ before and $45 \mathrm{~min}$ after positioning the micropipette. e. $\boldsymbol{f}$, Growth cone turning angle quantification. $\boldsymbol{g}$, Growth cone turning angle quantification in response to a gradient of Chariot complexed to VCA-GST or $\beta$-galactosidase as a control, delivered as above. $\boldsymbol{h}, \boldsymbol{k}$, Boundary assay for control (DMS0) and Arp2/3-inhibited (CK666) retina explants (left) with alternating lanes of laminin (black) and ephrin A2 + laminin (magenta) with axons stained with antibodies against the neuronal $\beta 3$-tubulin (green). $\boldsymbol{i}, \boldsymbol{j}, \boldsymbol{l}$, Show axons in close apposition to the ephrin A2 + laminin lanes at higher magnification.

In contrast to results on L1, Arp2/3 inhibition on laminin increased the actin retrograde flow along veils. Recently, Yang et al. (2012) reported similar findings and concluded that the branched actin network assembled by Arp2/3 can restrict the myosin II-dependent component of the actin retrograde flow. This suggests that during myosin II contractility a higher branch density in the actin network can slow its retrograde flow, presumably due to increased interactions with other cytoskeletal components like microtubules, other actin structures, or adhesions. As an alternative and complementary explanation for the increased actin retrograde flow, we found that Arp2/3 inhibition led to a reduction in the area and number of paxillin-GFP puncta per growth cone of 40 and $20 \%$, respectively (Fig. $8 a-f$ ). Therefore, Arp2/3-inhibited growth cones on laminin were less effective in engaging the "clutch" to slow the flow of actin filaments, leading to an increased retrograde flow rate. Notably, Arp2/3 inhibition in fibroblasts did not change adhesion size, only their organization relative to the leading edge (Wu et al., 2012). This discrepancy can be explained by the difference in cell type, substrate, and adhesions studied, since growth cone adhesions are round and small, versus the long linear focal adhesions seen in non-neuronal cells.
Why are adhesions smaller after Arp $2 / 3$ inhibition? It is known that Arp2/3 is recruited to nascent integrin adhesions (DeMali et al., 2002; Serrels et al., 2007) and that the actin retrograde flow can transfer myosin II-generated tension to promote adhesion growth and maturation (Galbraith et al., 2002; Choi et al., 2008; Wolfenson et al., 2011). Consequently, we propose that Arp2/3 inhibition reduces the chance for adhesions to interact with the overhead actin network because of its reduced branch density, in turn, leading to reduced tension on adhesions by the retrograde flow, which could reduce their maturation and/or stability. In agreement, Woo and Gomez (2006) showed that ROCK inhibition, a direct regulator of myosin II activity and actin retrograde flow, led to smaller and less stable growth cone adhesions on laminin.

Consistent with reports that actin polymerization is critical for guidance (Lanier et al., 1999; Marsick et al., 2010), growth cones exhibited reduced turning responses to chemoattractants (NGF or Netrin) and defective chemorepulsive responses (substrate-bound ephrin A2) on L1 during Arp2/3 inhibition. The reduced leading edge protrusion and the veil collapse during Arp2/3 inhibition would hamper growth cone turning toward an attractant. Similarly, when Arp2/3 was inhibited on a striped 
ephrin-A2 substrate, reduced protrusion at the growth cone side away from ephrin-A2 would cause defective evasion of ephrinA2. Additionally, we also showed that unilateral Arp2/3 activation with Chariot + VCA-GST was sufficient to trigger growth cone turning on L1. These results serve to highlight the central role the actin nucleator Arp2/3 plays during growth cone guidance on $\mathrm{L} 1$.

Nevertheless, performing the same attractive guidance assay on laminin during Arp2/3 inhibition caused no deficits. On the contrary, Arp2/3 inhibition led to increased turning responses during chemoattraction toward NGF. We can speculate on why these growth cones had a larger turning response. We know Arp2/3-inhibited growth cones on laminin have less and smaller adhesions, which would allow for faster dismantling and, hence, substrate detachment on the side opposite to the chemoattractant. This would allow growth cones to steer and follow the chemoattractant gradient more readily than control growth cones. Alternatively, larger turning responses of Arp2/3-inhibited growth cones could result from faster retrograde flow of actin. Neurotrophins increase the number of integrin adhesions of growth cones (Carlstrom et al., 2011). Therefore, NGF stimulation during turning could trigger the strengthening or formation of new adhesions closer to its source. In turn, these adhesions could harness the faster actin retrograde flow and convert it into a greater turning response. Even though Arp2/3 was inhibited, other actin nucleators like the formins and generation of barbed ends by ADF/cofilin severing activity could coordinate actin polymerization to steer growth cones toward the NGF source.

Similarly, Arp2/3 inhibition caused no defect on RGC growth cone response to avoid the ephrin A2 + laminin lanes on the boundary assay. Once again, other actin nucleators may be orchestrating actin filament polymerization to steer growth cones away from the ephrin A2 + laminin lanes. Alternatively, growth cones could steer away from the ephrin A2 + laminin lanes because of the reduced adhesivity they offer. It was shown that chemorepellent cues can dismantle integrin adhesions (Hines et al., 2010); therefore, in the boundary assay growth cones could remain on the laminin lanes because of their increased adhesivity, compared with the ephrin A2 + laminin ones. Also, supplemental to these results, we showed that delivery of the Chariot + VCA-GST complex, to unilaterally activate Arp $2 / 3$ on one side of the growth cone, failed to trigger turning on laminin. Therefore, we conclude that Arp2/3 is dispensable for an attractive guidance response to a soluble chemoattractant and for a repulsive guidance response to a membrane-bound chemorepellent, when growth cones migrate on laminin.

In conclusion, during development growth cones migrate in association with diverse substrates and respond to different guidance cues. In doing so, diverse signaling pathways are activated to regulate different $\mathrm{ABP}$ s to organize actin structures and modulate their dynamics to ensure that growth cones reach their targets. We probed the role of the actin nucleator Arp2/3 during growth cone motility and found it crucial for guidance on L1, but not on laminin. Previous studies have also shown the diversity of actin regulatory mechanisms in neuronal motility on different substrates. For example, Arp2/3-dependent actin structures were necessary for exocytosis and neuritogenesis on laminin, but not on PDL (poly-d-lysine), where exocytosis and neuritogenesis relied on Ena/VASP-dependent actin structures (Gupton and Gertler, 2010). In addition, ENA/VASP-null neurons cannot form neurites on PDL, but on laminin neurite formation by ENA/VASP-null neurons is normal (Dent et al., 2007).

\section{References}

Bentley D, Toroian-Raymond A (1986) Disoriented pathfinding by pioneer neurone growth cones deprived of filopodia by cytochalasin treatment. Nature 323:712-715. CrossRef Medline

Burden-Gulley SM, Payne HR, Lemmon V (1995) Growth cones are actively influenced by substrate-bound adhesion molecules. J Neurosci 15: 4370-4381. Medline

Butler B, Cooper JA (2009) Distinct roles for the actin nucleators Arp2/3 and hDial during NK-mediated cytotoxicity. Curr Biol 19:1886-1896. CrossRef Medline

Carlstrom LP, Hines JH, Henle SJ, Henley JR (2011) Bidirectional remodeling of beta1-integrin adhesions during chemotropic regulation of nerve growth. BMC Biol 9:82. CrossRef Medline

Chan CE, Odde DJ (2008) Traction dynamics of filopodia on compliant substrates. Science 322:1687-1691. CrossRef Medline

Choi CK, Vicente-Manzanares M, Zareno J, Whitmore LA, Mogilner A, Horwitz AR (2008) Actin and alpha-actinin orchestrate the assembly and maturation of nascent adhesions in a myosin II motor-independent manner. Nat Cell Biol 10:1039-1050. CrossRef Medline

Craig EM, Van Goor D, Forscher P, Mogilner A (2012) Membrane tension, myosin force, and actin turnover maintain actin treadmill in the nerve growth cone. Biophys J 102:1503-1513. CrossRef Medline

de la Torre JR, Höpker VH, Ming GL, Poo MM, Tessier-Lavigne M, Hemmati-Brivanlou A, Holt CE (1997) Turning of retinal growth cones in a netrin-1 gradient mediated by the netrin receptor DCC. Neuron 19:1211-1224. CrossRef Medline

Deiner MS, Kennedy TE, Fazeli A, Serafini T, Tessier-Lavigne M, Sretavan DW (1997) Netrin-1 and DCC mediate axon guidance locally at the optic disc: loss of function leads to optic nerve hypoplasia. Neuron 19: 575-589. CrossRef Medline

DeMali KA, Barlow CA, Burridge K (2002) Recruitment of the Arp2/3 complex to vinculin: coupling membrane protrusion to matrix adhesion. J Cell Biol 159:881-891. CrossRef Medline

Dent EW, Kwiatkowski AV, Mebane LM, Philippar U, Barzik M, Rubinson DA, Gupton S, Van Veen JE, Furman C, Zhang J, Alberts AS, Mori S, Gertler FB (2007) Filopodia are required for cortical neurite initiation. Nat Cell Biol 9:1347-1359. CrossRef Medline

Dent EW, Gupton SL, Gertler FB (2011) The growth cone cytoskeleton in axon outgrowth and guidance. Cold Spring Harb Perspect Biol 3: pii: a001800. CrossRef

Di Nardo A, Cicchetti G, Falet H, Hartwig JH, Stossel TP, Kwiatkowski DJ (2005) Arp $2 / 3$ complex-deficient mouse fibroblasts are viable and have normal leading-edge actin structure and function. Proc Natl Acad Sci U S A 102:16263-16268. CrossRef Medline

Duleh SN, Welch MD (2010) WASH and the Arp2/3 complex regulate endosome shape and trafficking. Cytoskeleton (Hoboken) 67:193-206. CrossRef

Galbraith CG, Yamada KM, Sheetz MP (2002) The relationship between force and focal complex development. J Cell Biol 159:695-705. CrossRef Medline

Giancotti FG, Ruoslahti E (1999) Integrin signaling. Science 285:10281032. CrossRef Medline

Goley ED, Welch MD (2006) The ARP2/3 complex: an actin nucleator comes of age. Nat Rev Mol Cell Biol 7:713-726. CrossRef Medline

Gomez TM, Letourneau PC (2013) Actin dynamics in growth cone motility and navigation. J Neurochem. Advance online publication. Retrieved October 24, 2013. doi:10.1111/jnc.12506.

Gupton SL, Gertler FB (2010) Integrin signaling switches the cytoskeletal and exocytic machinery that drives neuritogenesis. Dev Cell 18:725-736. CrossRef Medline

Henson JH, Svitkina TM, Burns AR, Hughes HE, MacPartland KJ, Nazarian R, Borisy GG (1999) Two components of actin-based retrograde flow in sea urchin coelomocytes. Mol Biol Cell 10:4075-4090. CrossRef Medline

Hines JH, Abu-Rub M, Henley JR (2010) Asymmetric endocytosis and remodeling of betal-integrin adhesions during growth cone chemorepulsion by MAG. Nat Neurosci 13:829-837. CrossRef Medline

Hüfner K, Higgs HN, Pollard TD, Jacobi C, Aepfelbacher M, Linder S (2001) The verprolin-like central (vc) region of Wiskott-Aldrich syndrome protein induces Arp2/3 complex-dependent actin nucleation. J Biol Chem 276:35761-35767. CrossRef Medline

Kamiguchi H, Lemmon V (1997) Neural cell adhesion molecule L1: signal- 
ing pathways and growth cone motility. J Neurosci Res 49:1-8. CrossRef Medline

Korobova F, Svitkina T (2008) Arp2/3 complex is important for filopodia formation, growth cone motility, and neuritogenesis in neuronal cells. Mol Biol Cell 19:1561-1574. CrossRef Medline

Kwiatkowski AV, Rubinson DA, Dent EW, Edward van Veen J, Leslie JD, Zhang J, Mebane LM, Philippar U, Pinheiro EM, Burds AA, Bronson RT, Mori S, Fässler R, Gertler FB (2007) Ena/VASP Is Required for neuritogenesis in the developing cortex. Neuron 56:441-455. CrossRef Medline

Lanier LM, Gates MA, Witke W, Menzies AS, Wehman AM, Macklis JD, Kwiatkowski D, Soriano P, Gertler FB (1999) Mena is required for neurulation and commissure formation. Neuron 22:313-325. CrossRef Medline

Lin CH, Espreafico EM, Mooseker MS, Forscher P (1996) Myosin drives retrograde F-actin flow in neuronal growth cones. Neuron 16:769-782. CrossRef Medline

Liu RY, Schmid RS, Snider WD, Maness PF (2002) NGF enhances sensory axon growth induced by laminin but not by the $\mathrm{L} 1 \mathrm{cell}$ adhesion molecule. Mol Cell Neurosci 20:2-12. CrossRef Medline

Machesky LM, Insall RH (1998) Scar1 and the related Wiskott-Aldrich syndrome protein, WASP, regulate the actin cytoskeleton through the Arp2/3 complex. Curr Biol 8:1347-1356. CrossRef Medline

Marsick BM, Letourneau PC (2011) Labeling F-actin barbed ends with rhodamine-actin in permeabilized neuronal growth cones. J Vis Exp 17: pii: 2409. CrossRef

Marsick BM, Flynn KC, Santiago-Medina M, Bamburg JR, Letourneau PC (2010) Activation of ADF/cofilin mediates attractive growth cone turning toward nerve growth factor and netrin-1. Dev Neurobiol 70:565-588. CrossRef Medline

Marsick BM, San Miguel-Ruiz JE, Letourneau PC (2012) Activation of ezrin/radixin/moesin mediates attractive growth cone guidance through regulation of growth cone actin and adhesion receptors. J Neurosci 32: 282-296. CrossRef Medline

McLoon SC, McLoon LK, Palm SL, Furcht LT (1988) Transient expression of laminin in the optic nerve of the developing rat. J Neurosci 8:19811990. Medline

Medeiros NA, Burnette DT, Forscher P (2006) Myosin II functions in actinbundle turnover in neuronal growth cones. Nat Cell Biol 8:215-226. Medline

Merrifield CJ, Qualmann B, Kessels MM, Almers W (2004) Neural Wiskott Aldrich Syndrome Protein (N-WASP) and the Arp2/3 complex are recruited to sites of clathrin-mediated endocytosis in cultured fibroblasts. Eur J Cell Biol 83:13-18. CrossRef Medline

Mongiu AK, Weitzke EL, Chaga OY, Borisy GG (2007) Kinetic-structural analysis of neuronal growth cone veil motility. J Cell Sci 120:1113-1125. CrossRef Medline

Mukai Y, Iwaya K, Ogawa H, Mukai K (2005) Involvement of Arp2/3 complex in MCP-1-induced chemotaxis. Biochem Biophys Res Commun 334: 395-402. CrossRef Medline

Mullins RD, Stafford WF, Pollard TD (1997) Structure, subunit topology, and actin-binding activity of the Arp2/3 complex from Acanthamoeba. J Cell Biol 136:331-343. CrossRef Medline

Nakamura Y, Lee S, Haddox CL, Weaver EJ, Lemmon VP (2010) Role of the cytoplasmic domain of the L1 cell adhesion molecule in brain development. J Comp Neurol 518:1113-1132. CrossRef Medline

Nolen BJ, Tomasevic N, Russell A, Pierce DW, Jia Z, McCormick CD, Hartman J, Sakowicz R, Pollard TD (2009) Characterization of two classes of small molecule inhibitors of Arp2/3 complex. Nature 460:1031-1034. CrossRef Medline

Norris AD, Dyer JO, Lundquist EA (2009) The Arp2/3 complex, UNC-115/ abLIM, and UNC-34/Enabled regulate axon guidance and growth cone filopodia formation in Caenorhabditis elegans. Neural Dev 4:38. CrossRef Medline

Pollard TD (2007) Regulation of actin filament assembly by Arp2/3 complex and formins. Annu Rev Biophys Biomol Struct 36:451-477. CrossRef Medline

Serrels B, Serrels A, Brunton VG, Holt M, McLean GW, Gray CH, Jones GE, Frame MC (2007) Focal adhesion kinase controls actin assembly via a FERM-mediated interaction with the Arp $2 / 3$ complex. Nat Cell Biol 9:1046-1056. CrossRef Medline

Shakir MA, Jiang K, Struckhoff EC, Demarco RS, Patel FB, Soto MC, Lundquist EA (2008) The Arp2/3 activators WAVE and WASP have distinct genetic interactions with Rac GTPases in Caenorhabditis elegans axon guidance. Genetics 179:1957-1971. CrossRef Medline

Shimada T, Toriyama M, Uemura K, Kamiguchi H, Sugiura T, Watanabe N, Inagaki N (2008) Shootin1 interacts with actin retrograde flow and L1CAM to promote axon outgrowth. J Cell Biol 181:817-829. CrossRef Medline

Sigal YJ, Quintero OA, Cheney RE, Morris AJ (2007) Cdc42 and ARP2/3independent regulation of filopodia by an integral membrane lipidphosphatase-related protein. J Cell Sci 120:340-352. CrossRef Medline

Spillane M, Ketschek A, Jones SL, Korobova F, Marsick B, Lanier L, Svitkina T, Gallo G (2011) The actin nucleating Arp2/3 complex contributes to the formation of axonal filopodia and branches through the regulation of actin patch precursors to filopodia. Dev Neurobiol 71:747-758. CrossRef Medline

Strasser GA, Rahim NA, VanderWaal KE, Gertler FB, Lanier LM (2004) Arp2/3 is a negative regulator of growth cone translocation. Neuron 43: 81-94. CrossRef Medline

Suraneni P, Rubinstein B, Unruh JR, Durnin M, Hanein D, Li R (2012) The Arp2/3 complex is required for lamellipodia extension and directional fibroblast cell migration. J Cell Biol 197:239-251. CrossRef Medline

Suter DM, Errante LD, Belotserkovsky V, Forscher P (1998) The Ig superfamily cell adhesion molecule, apCAM, mediates growth cone steering by substrate-cytoskeletal coupling. J Cell Biol 141:227-240. CrossRef Medline

Svitkina TM, Bulanova EA, Chaga OY, Vignjevic DM, Kojima S, Vasiliev JM, Borisy GG (2003) Mechanism of filopodia initiation by reorganization of a dendritic network. J Cell Biol 160:409-421. CrossRef Medline

van der Gucht J, Paluch E, Plastino J, Sykes C (2005) Stress release drives symmetry breaking for actin-based movement. Proc Natl Acad Sci U S A 102:7847-7852. CrossRef Medline

Wolfenson H, Bershadsky A, Henis YI, Geiger B (2011) Actomyosingenerated tension controls the molecular kinetics of focal adhesions. J Cell Sci 124:1425-1432. CrossRef Medline

Woo S, Gomez TM (2006) Racl and RhoA promote neurite outgrowth through formation and stabilization of growth cone point contacts. J Neurosci 26:1418-1428. CrossRef Medline

Wu C, Asokan SB, Berginski ME, Haynes EM, Sharpless NE, Griffith JD, Gomez SM, Bear JE (2012) Arp2/3 is critical for lamellipodia and response to extracellular matrix cues but is dispensable for chemotaxis. Cell 148:973-987. CrossRef Medline

Yang Q, Zhang XF, Pollard TD, Forscher P (2012) Arp2/3 complexdependent actin networks constrain myosin II function in driving retrograde actin flow. J Cell Biol 197:939-956. CrossRef Medline 